九州大学学術情報リポジトリ

Kyushu University Institutional Repository

\title{
Power Optimization by Datapath Width Adjustment
}

Yasuura, Hiroto

Faculty of Information Science and Electrical Engineering, Kyushu University | System LSI

Research Center

ht tp://hdl. hand le. net/2324/9119

出版情報 : SLRC プレゼンテーション，2005-10-19

バージョン：

権利関係 : 


\section{POWER OPTIMIZATION BY DATAPATH WIDTH ADJUSTMENT}

\section{Hiroto Yasuura \\ System LSI Research Center \\ Kyushu University}




\section{POWER OPTIMIZATION BY DATAPATH WIDTH}

ADJUSTMENT

- Introduction

- Datapath Width and Power Consumption

- Soft Core Processor

- Memory Width Optimization

- Shifted Computation

- Value-Based Clock Gating

- Conclusion 


\section{Low Energy SoC}

- Increase of \# of Transistors in a Chip

- Dynamic Power Consumption (\# of Switching)

- Static Power Consumption (Leakage Power)

- Memory, Analog, RF and MEMS

- Increase of Clock Frequency

- Applications in Mobile Devices

- Battery Driven Systems

- Battery Free Systems (RF ID Tags, Sensor Networks)

- Energy Limitation of the Earth 


\section{Ultra Low Power Design Technologies}

$\mu \mathrm{W}$ Class

mW Class

W Class

4

Wireless Power Supply Self Power Generation
Battery Driven

Air Cooling
Sensor Network

RF ID Tag

IC Card
Mobile Device

Automobile
High Performance

Computing

PC, DTV, DVD 


\section{Techniques for Energy Reduction}

- Dynamic Energy Reduction

- Voltage Scaling (Dynamic Voltage Scheduling)

- Reduction of Capacitance

- Reduction of \# of Switchings

- Clock Gating

- Datapath Width Adjustment

- Static Energy (Leakage) Reduction

- Threshold Control

- New Device/Circuit Structure

- Power Supply Gating

- Datapath Width Adjustment 


\section{POWER OPTIMIZATION BY DATAPATH WIDTH}

ADJUSTMENT

- Introduction

- Datapath Width and Power Consumption

- Soft Core Processor

- Memory Width Optimization

- Shifted Computation

- Value-Based Clock Gating

- Conclusion 


\section{Datapath Width and Power Consumption}

- The datapath width of the system

- Buses, ALUs, Registers, Memories...

- Determined by system designers or standard parts.

- Each application requires different accuracy of computation.

- Specifications of input/output signals (Requirement of Quality)

- Requests from algorithms (Requirement of Accuracy)

- Required datapath width is different from the width of HW.

- Larger datapath width for small bit-width data

- Wasteful dynamic power in switching of the extra bits on the datapath.

- Extra leakage power consumption 


\section{MPEG-2 Video Decoder}

Size of Program : 6275 lines (written in C)

Varable size
analusicresult.
ariable Size Analysis Results

\section{2 bits Variables}

384 int type

variables

\begin{tabular}{|c|c|}
\hline 8 bits & 9 \\
\hline 9 bits & 7 \\
\hline 10 bits & 3 \\
\hline 11 bits & 6 \\
\hline 12 bits & 17 \\
\hline 13 bits & 0 \\
\hline 14 bits & 46 \\
\hline 15 bits & 2 \\
\hline 16 bits & 39 \\
\hline
\end{tabular}

\begin{tabular}{|l|l|}
\hline 24 bits & 13 \\
\hline 25 bits & 0 \\
\hline 26 bits & 2 \\
\hline 27 bits & 4 \\
\hline 28 bits & 3 \\
\hline 29 bits & 3 \\
\hline 30 bits & 7 \\
\hline 31 bits & 0 \\
\hline 32 bits & 5 \\
\hline
\end{tabular}

$x * y: x$ is \# of elements $y$ is \# of arrays 


\section{Variable data-path Architecture}

- Data-path which can be activated in 4-bit width.

- Variables A:16bits, B:4bits, C:12bits Ordinary Data-path Variable Data-path

A 1100111111000011 A 1100111111000011

B 0000000000000100 B 1100111111000100

C 1111111100101111 C 1100111100101111

\# of SW $=23 \quad$ \# of $S W=9$ 


\section{Experimental Results}

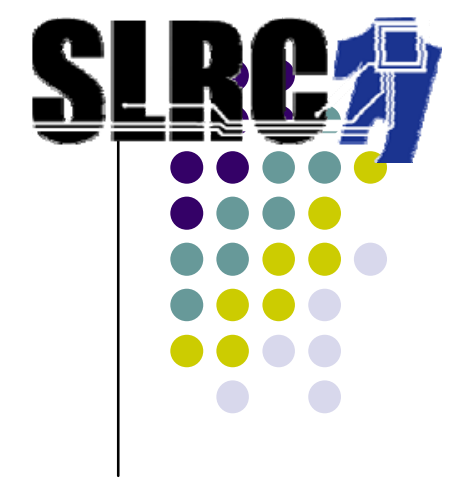

- Program

- Split (File Split) and Sort in Unix

- Architecture

- Variable Data-path Architecture Processor: VDP

- 32bit RISC Processor: Fixed

\begin{tabular}{l|llll} 
& \multicolumn{2}{|c}{ \# of Gates Delay } & Split & \multicolumn{1}{c}{ Sort } \\
\hline VDP & 3,478 & $28.46 \mathrm{~ns}$ & $60.57 \mathrm{~mW}$ & $69.18 \mathrm{~mW}$ \\
Fixed & 3,199 & $27.47 \mathrm{~ns}$ & $72.39 \mathrm{~mW}$ & $96.05 \mathrm{~mW}$ \\
Power Reduction & & $-16.32 \%$ & $-27.98 \%$
\end{tabular}




\section{POWER OPTIMIZATION BY DATAPATH}

WIDTH ADJUSTMENT

- Introduction

- Datapath Width and Power Consumption

- Soft Core Processor

- Memory Width Optimization

- Shifted Computation

- Value-Based Clock Gating

- Conclusion 


\section{Reducing the lower bits of datapath}

Changing Computation Quality

- When the accuracy of computation can be reduced, system designer can reduce the lower bits of variables.

Program

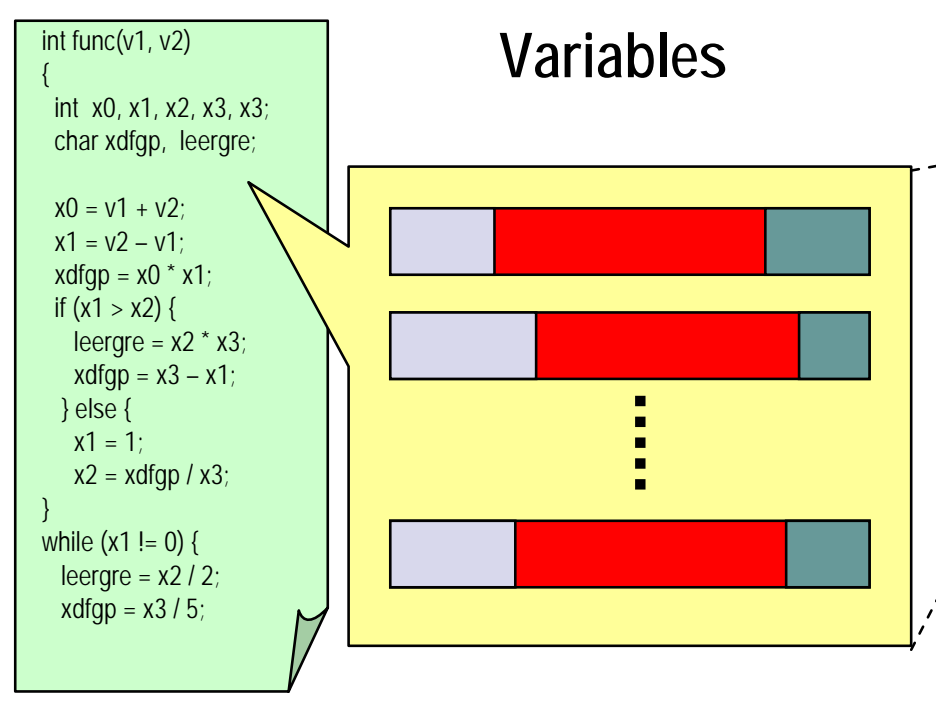

Datapath

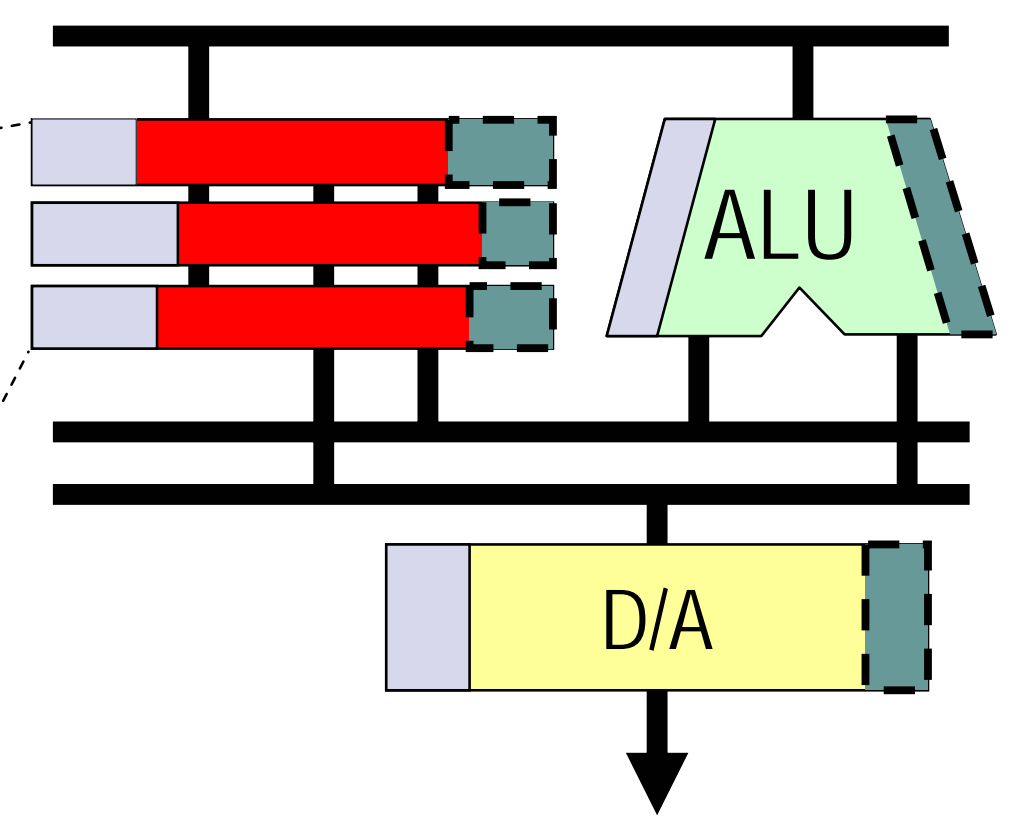

Output 


\section{Reducing the upper bits of datapath}

No Chang in Quality

- It is difficult to know the range of the variables.

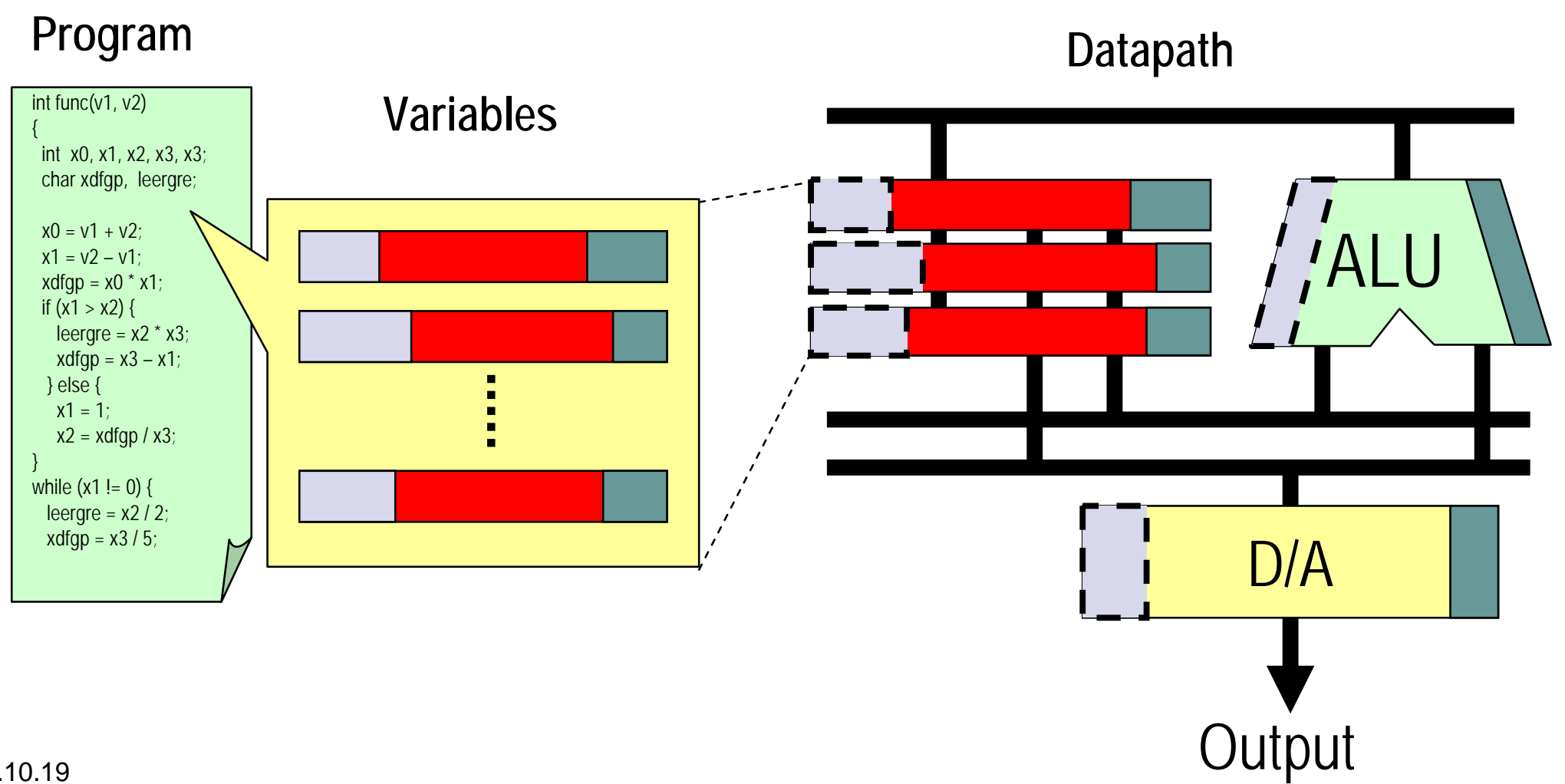




\section{Result of Variable Size Analysis}

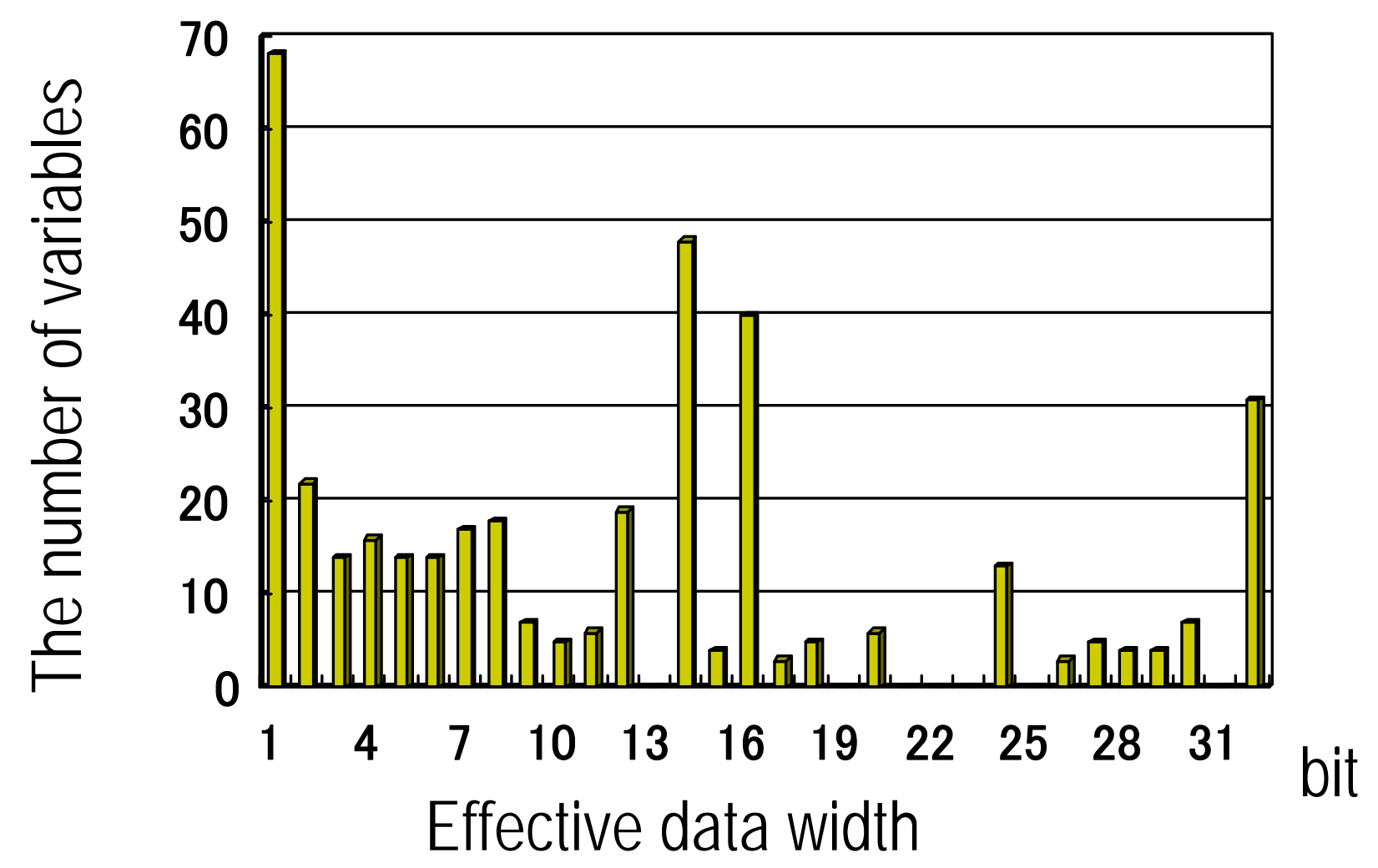

MPEG-2 video decoder 


\section{Variable Size Analysis}

- Concepts

- Examine the range of variables and find the required bit-width for each variables.

- By analyzing required bit-width of variables, the datapath width can be optimized for power minimization

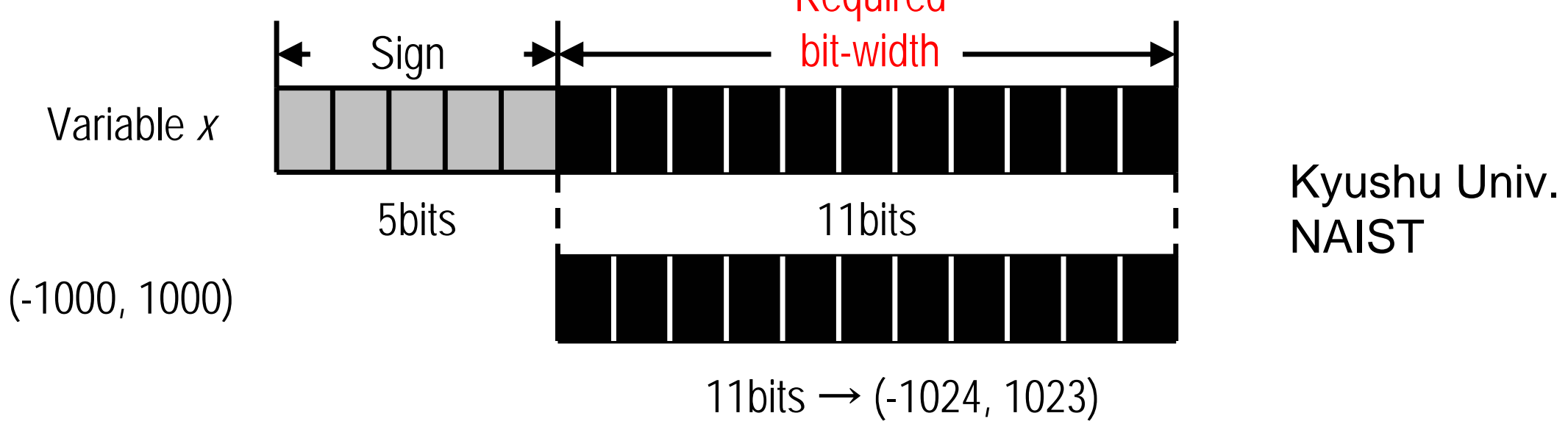

$\underset{05510.19}{\text { MPEG2 Decoder: }} \frac{\text { Variable Size Analysis Results }}{32 \text { bits Variables }} * 100=\underline{35 \%}$ 


\section{Soft-core Processor}

- Parameterized core processor

- Design parameters

- the datapath width

- the number of general registers

- Instruction set

- Data/instruction memory space

- Logic and layout synthesis tools

- Retargetable compiler

k IP of customized core processor 


\section{Valen-C and a Retargetable Compiler}

- Valen-C

- Programmers can specify the effective bit width for each variable.

$$
\text { int20 } x, y, z
$$

- The semantics of the program can be independent from processor architecture.

- Retargetable compiler

- Processor Definition + Valen-C Program

$\Rightarrow$ Assembly code for the processor 


\section{Datapath Width and Data Memory}

\author{
Valen-C Program \\ int12 $x$; \\ int20 y; \\ int24 z;
}

20-bit processor

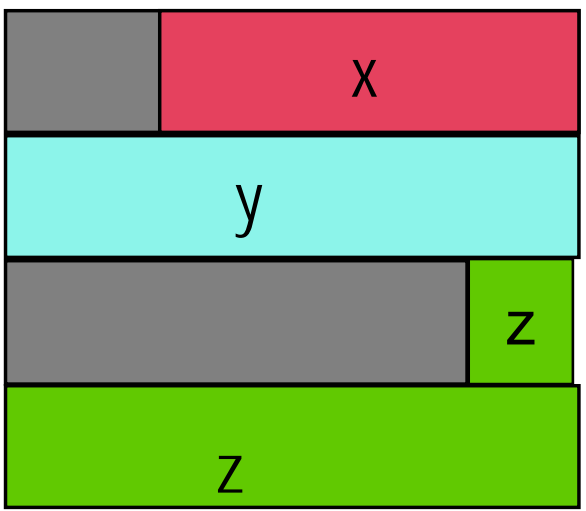

unused: 24 bits total: 80 bits

unused bits 12-bit processor

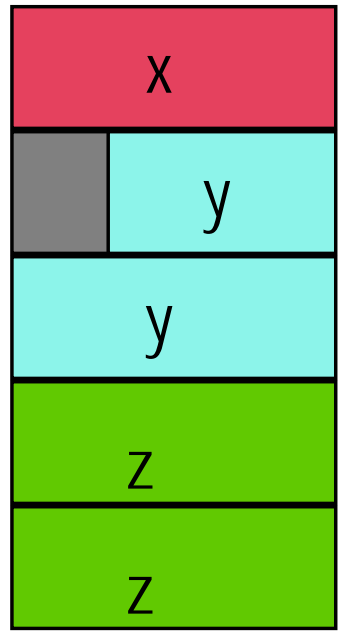

unused: 4 bits total: 60 bits 


\section{A Simple Example}

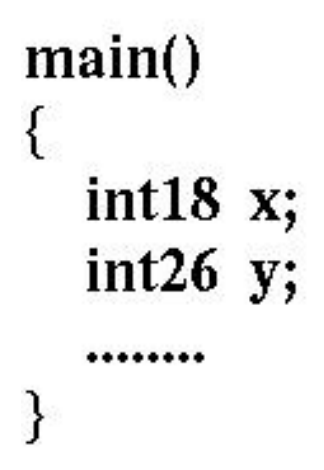

32 bits $\times 2$ words $=64$ bits

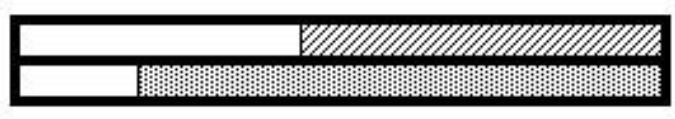

26 bits $\times 2$ words $=52$ bits
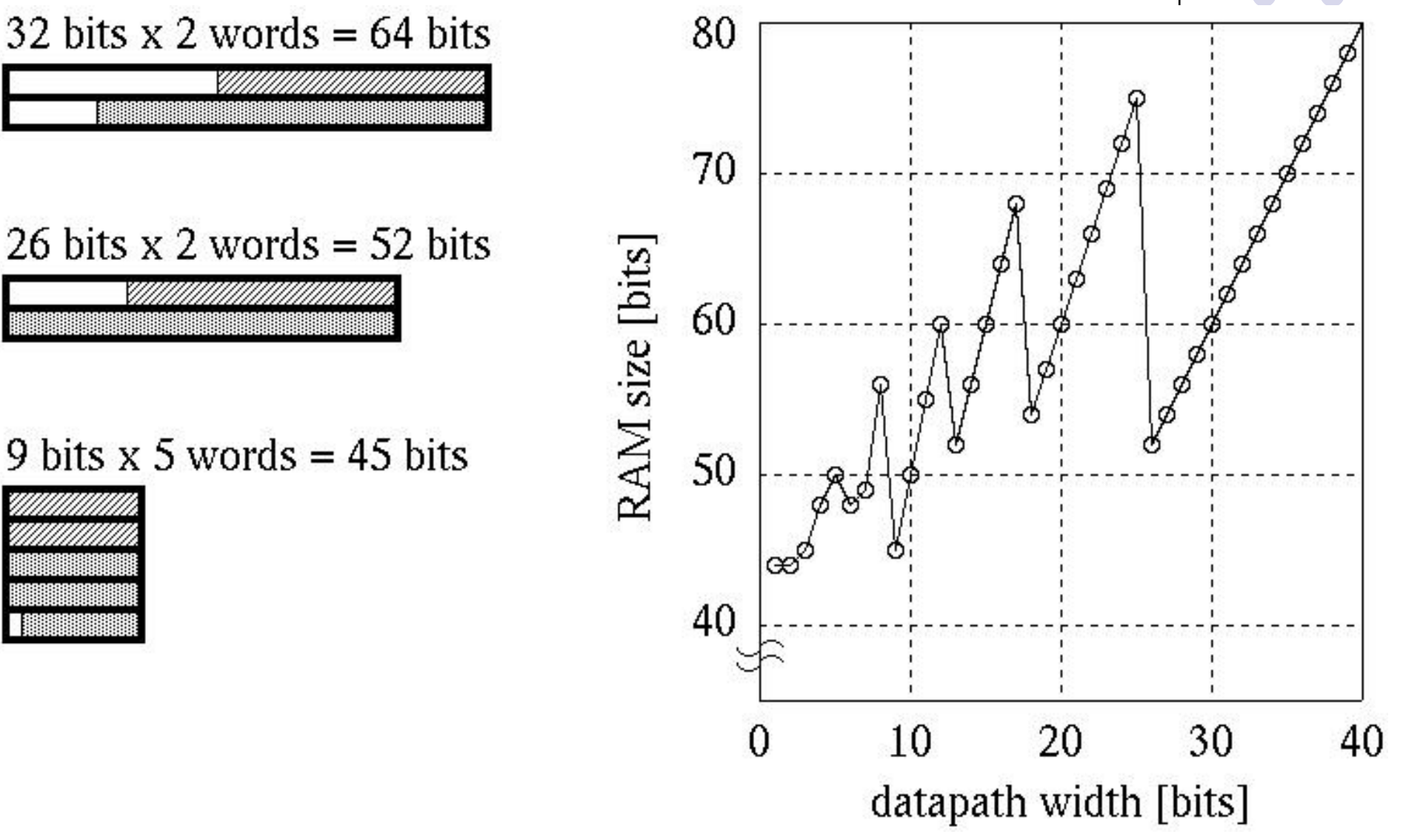


\section{Trade-off between Area and Performance}

int20 $\mathrm{x}, \mathrm{y}, \mathrm{z}$;

$\mathbf{z}=\mathbf{x}+\mathbf{y}$;

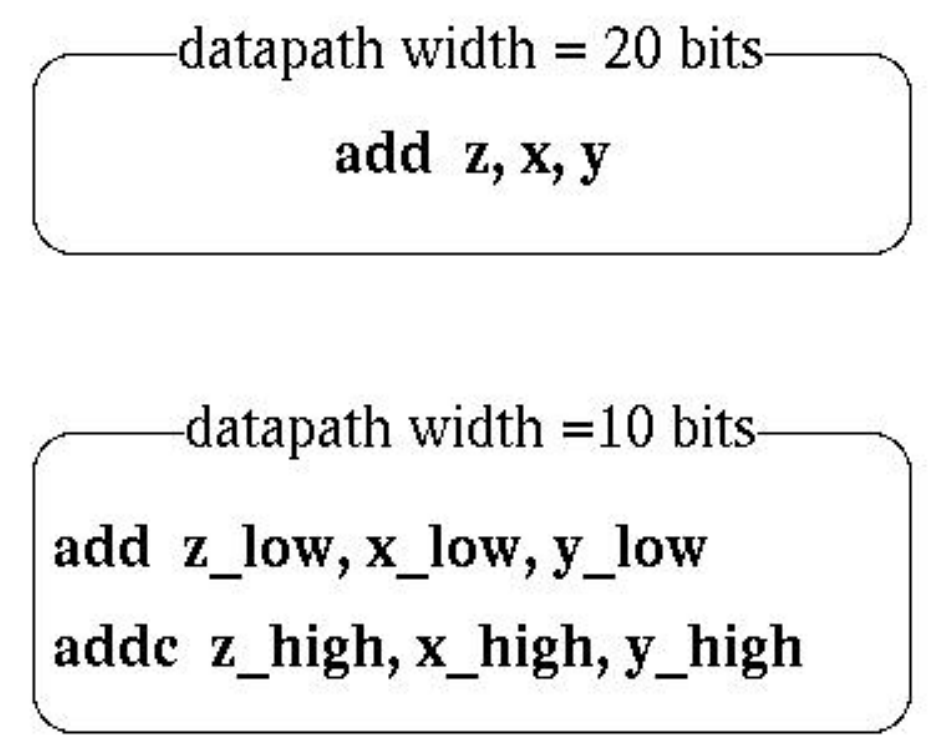

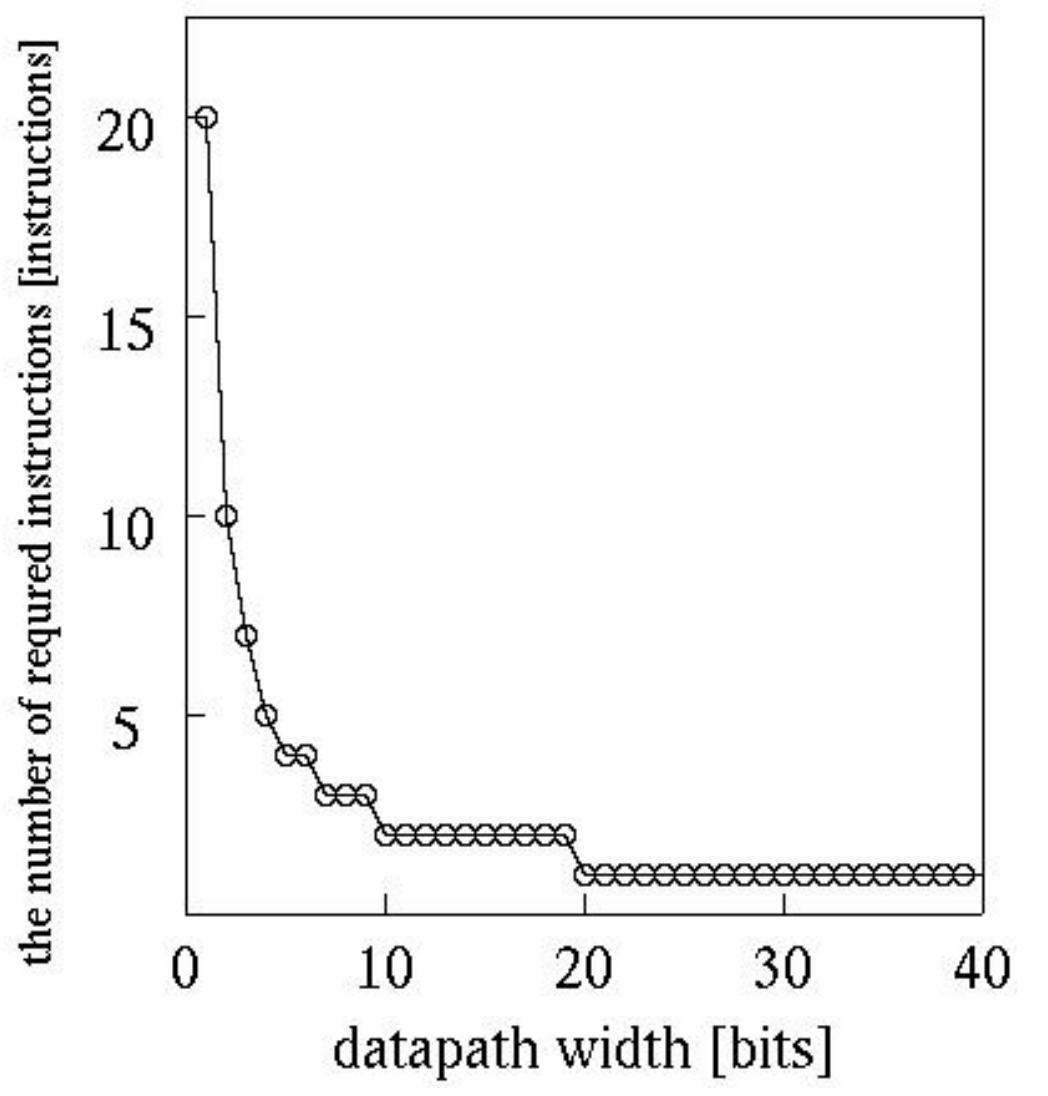


Design Flow of Datapath Width Optimization

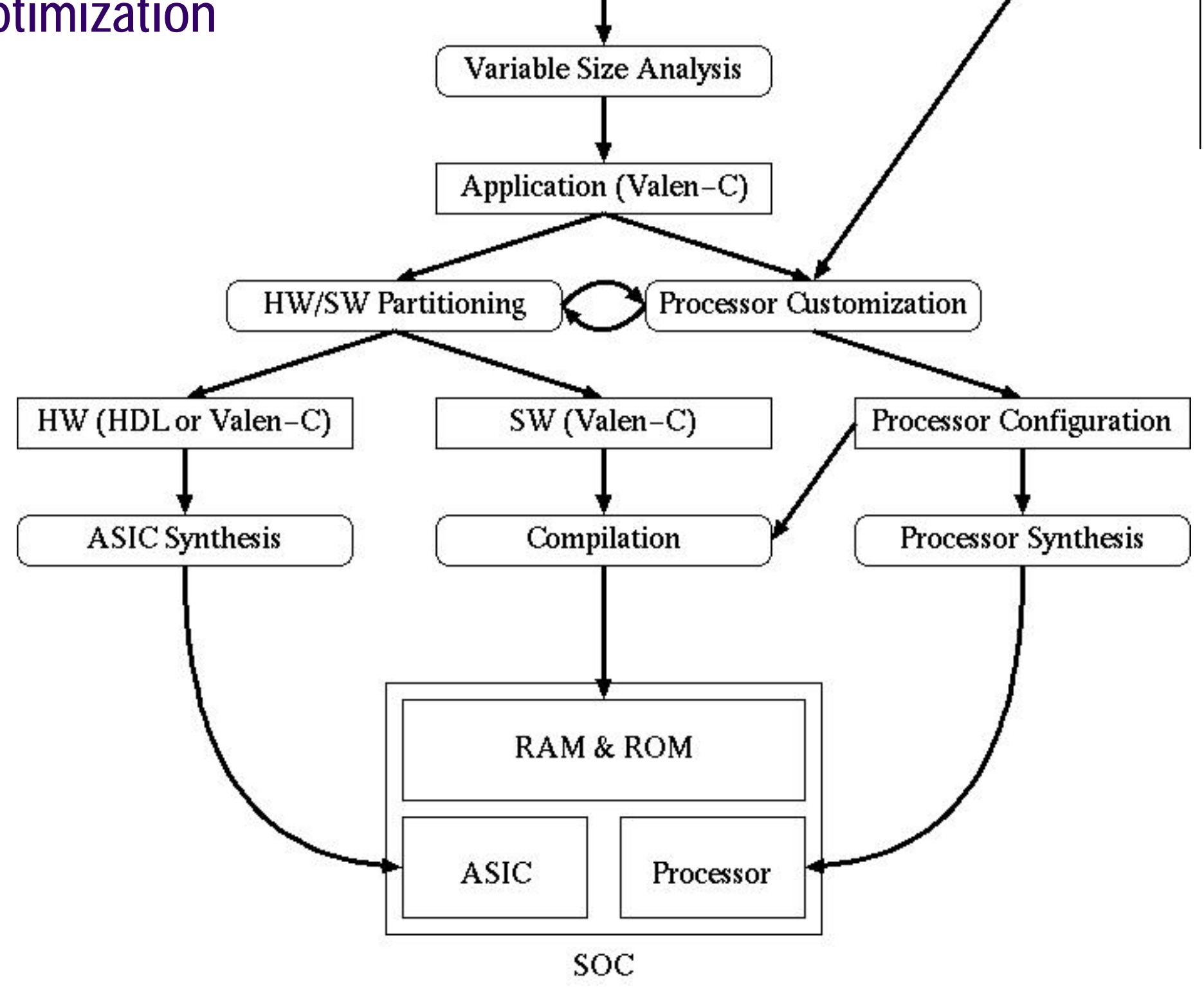




\section{Valen-C Compiler}

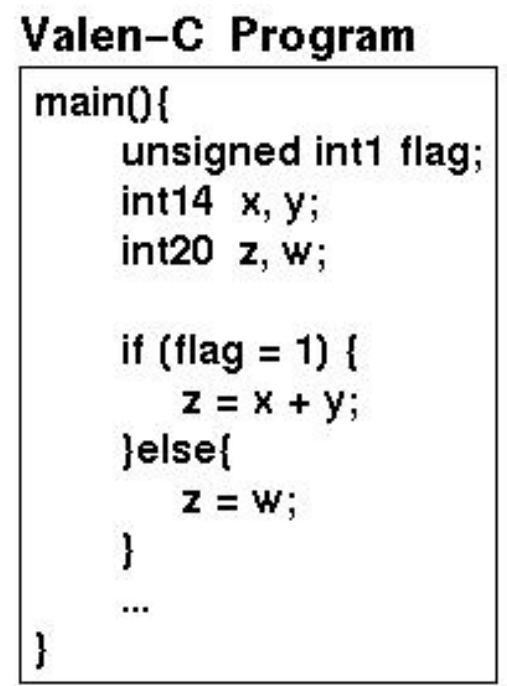

\section{Machine Description File}

Datapath width:10bits

short : 5 bits

int $: 10$ bits

long : 20 bits

long long : 30 bits

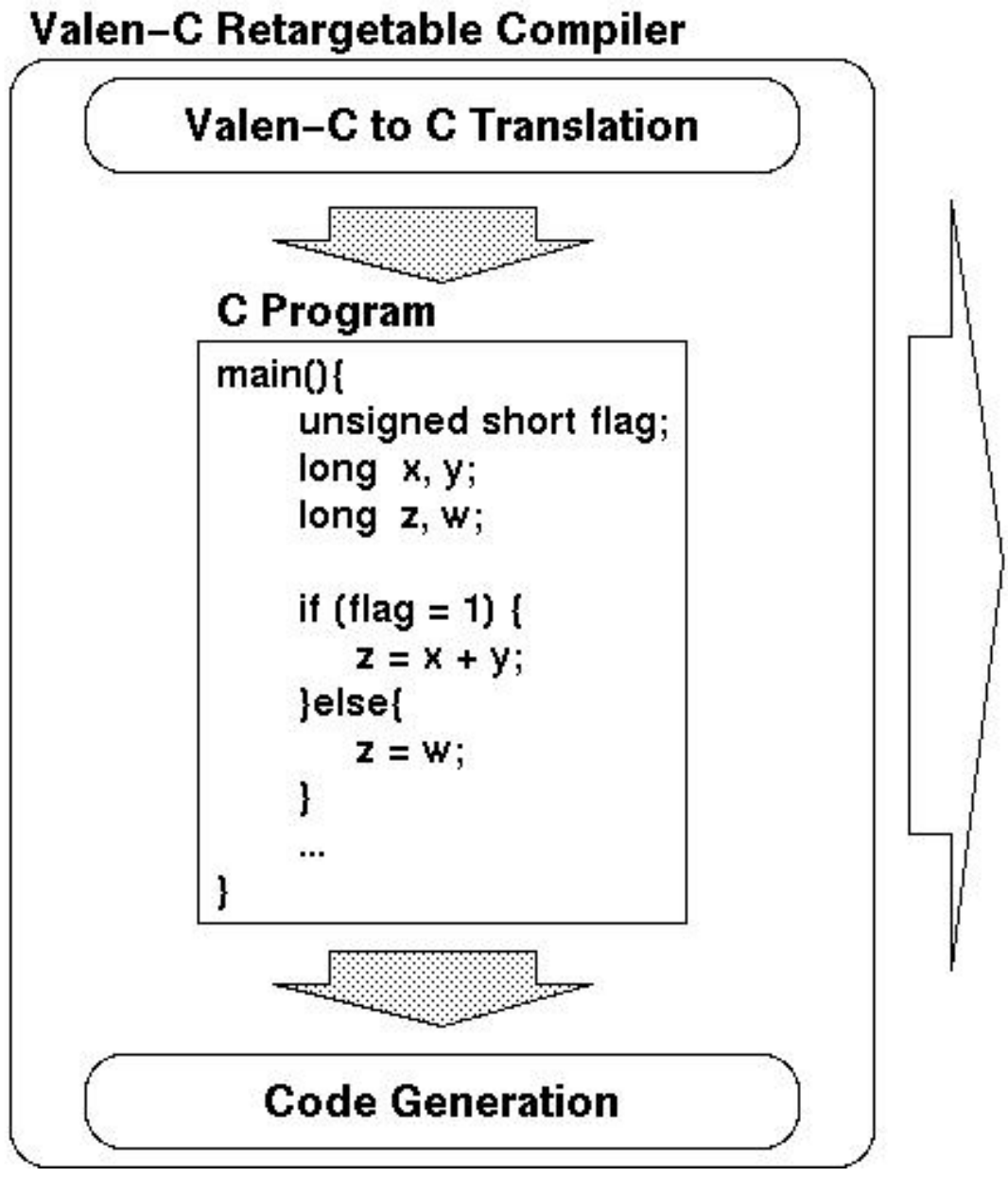

\section{Assembly Code}

seq tmp, flag, 1

beqz tmp, L1

add $\mathrm{zl}, \mathrm{xl}, \mathrm{yl}$;

addc zu, xu, yu;

jmp L2:

L1: move zl, wl;

move zu wu;

L2:

zl : lower bits of z

$\mathbf{z u}$ : upper bits of $\mathbf{z}$

seq : set conditinal if two operands are equal each other

addc : add with carry

beqz : branch equal zero 


\section{Flow of Valen-C Compiler}

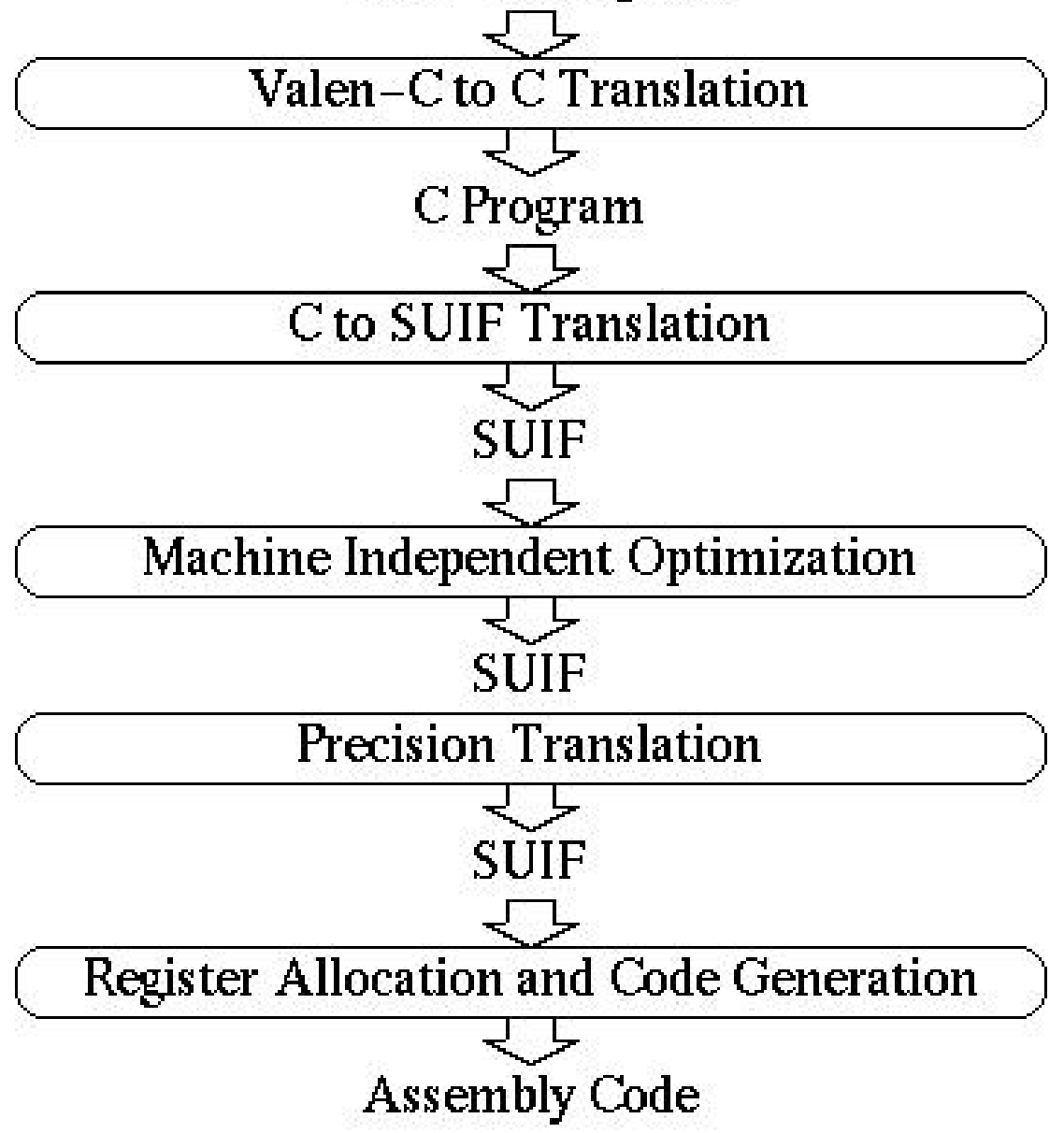




\section{Datapath Width Adjustment for a Calculator}

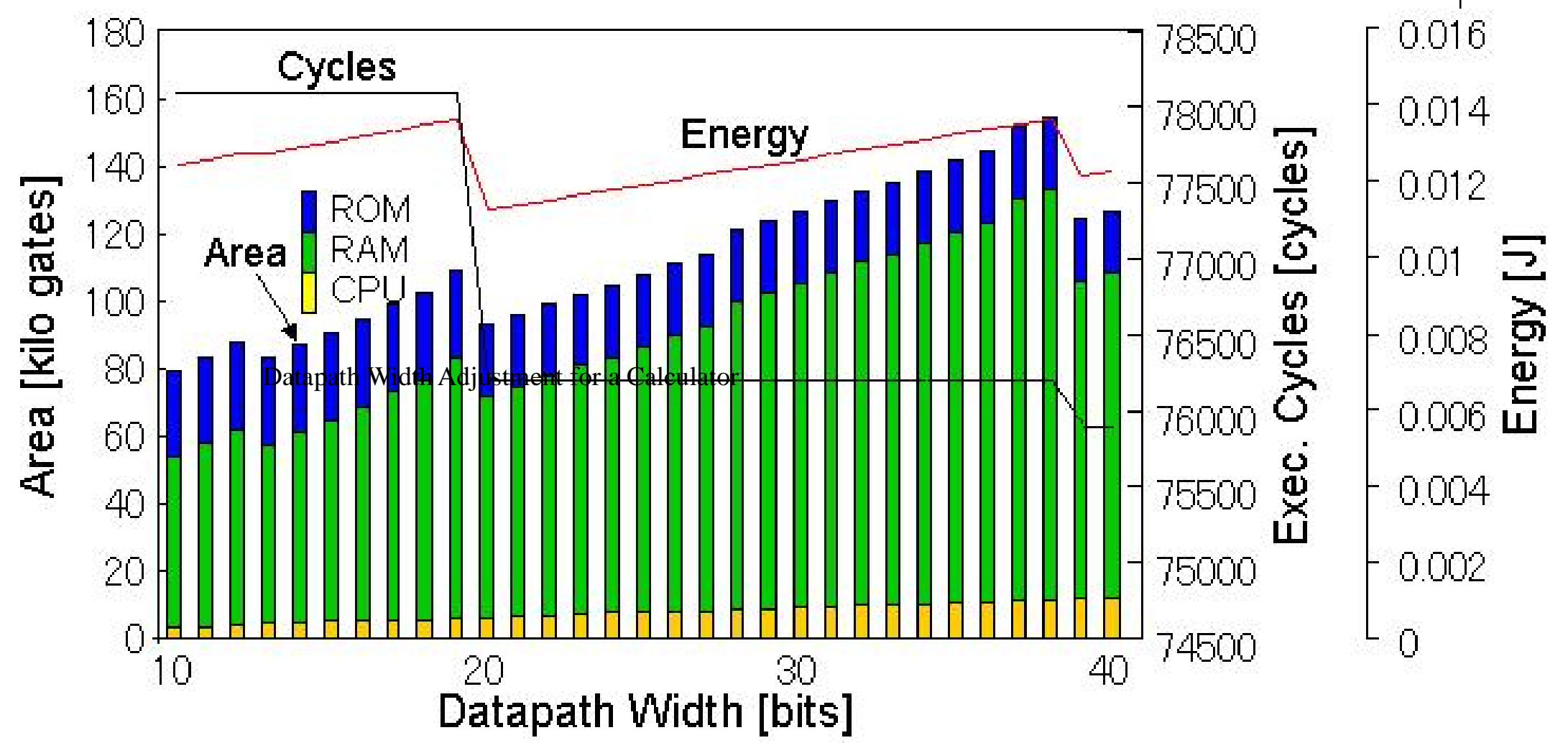


Datapath Width Adjustment for Lempel-Ziv encoder/decoder

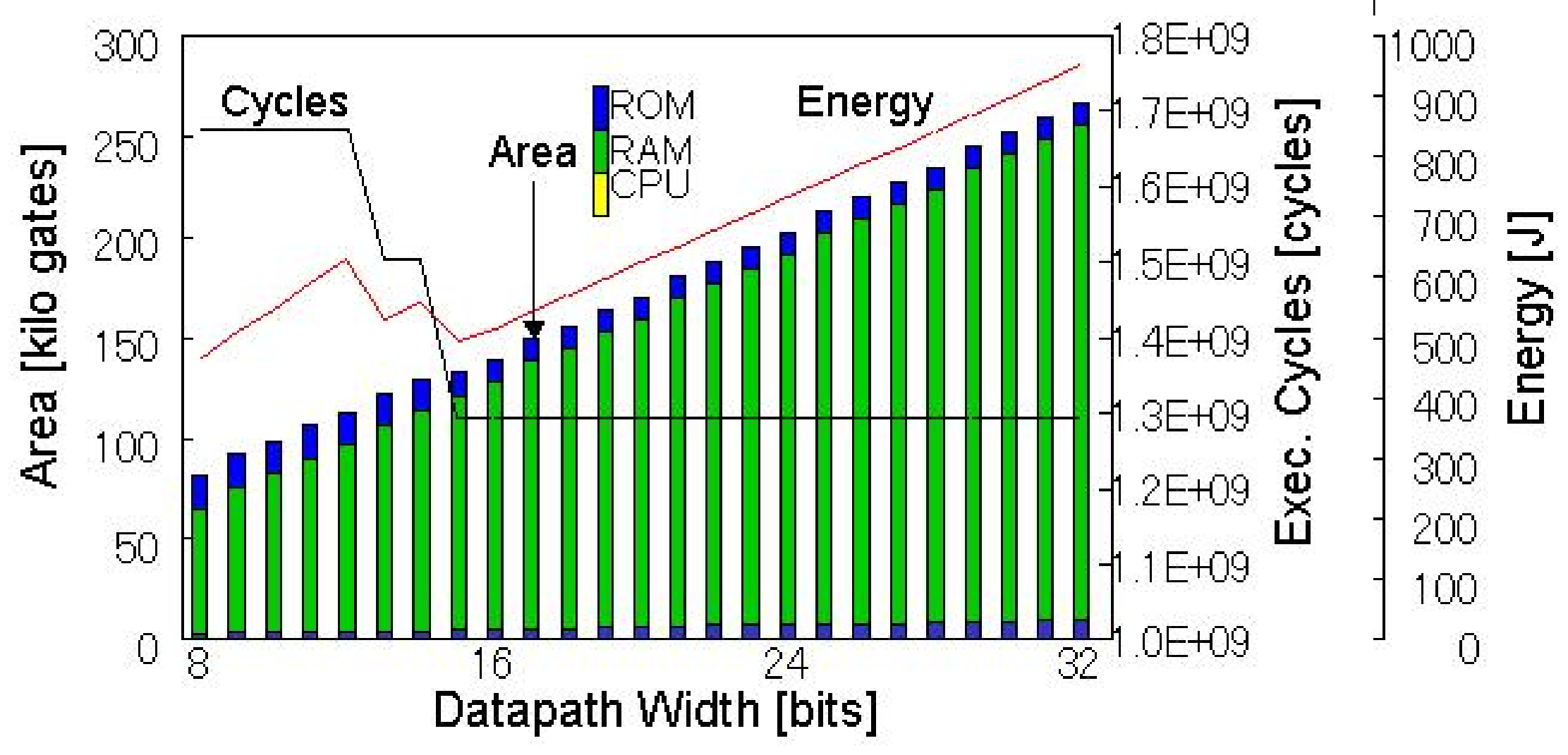




\section{Datapath Width Adjustment for ADPCM encoder}

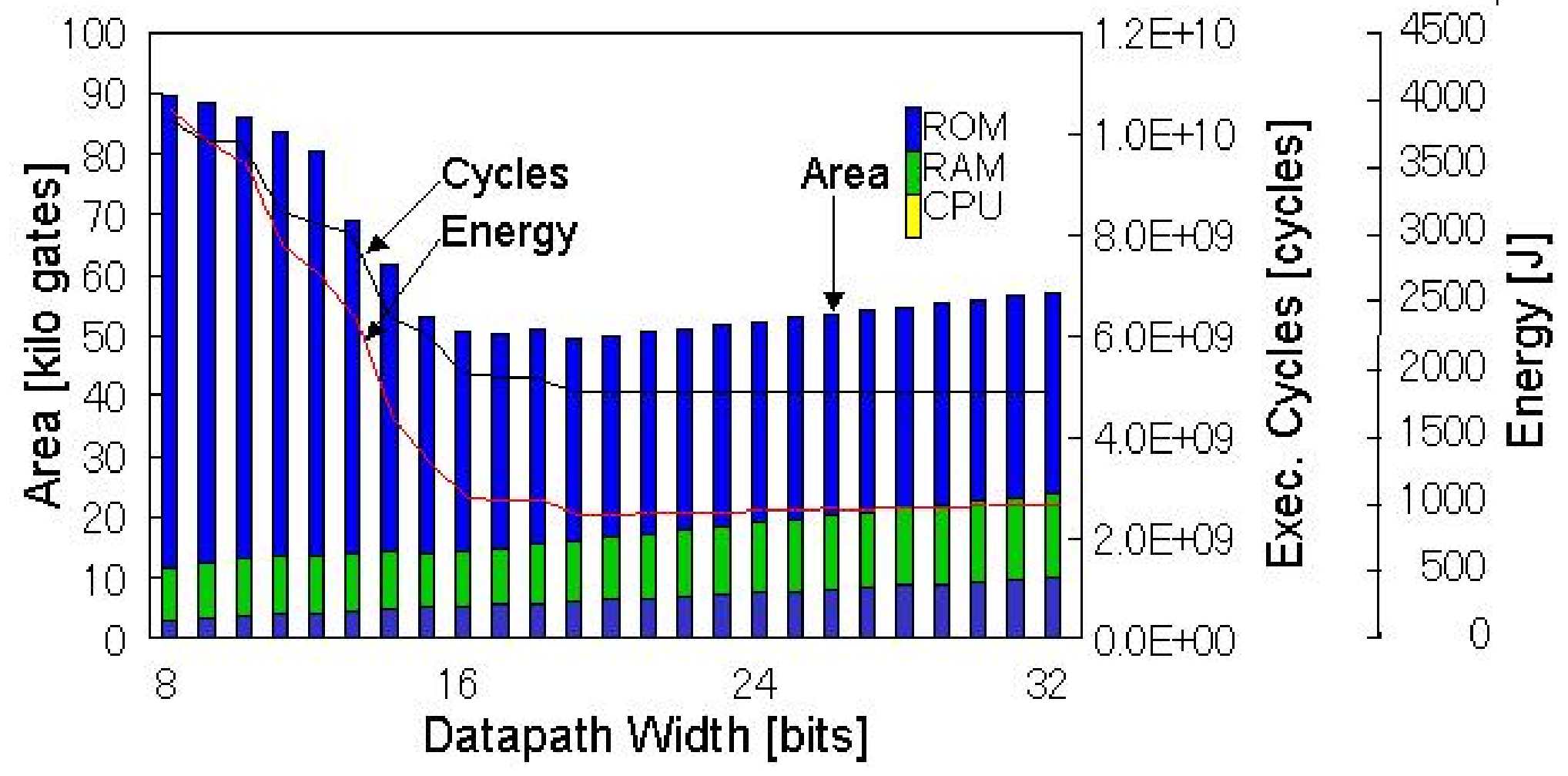




\section{Example of \\ Datapath Width Optimization}

ADPCM decoder

[Variables]
bufferstep
inputbuffer
index
vpdiff
valpred
step
delta
sign
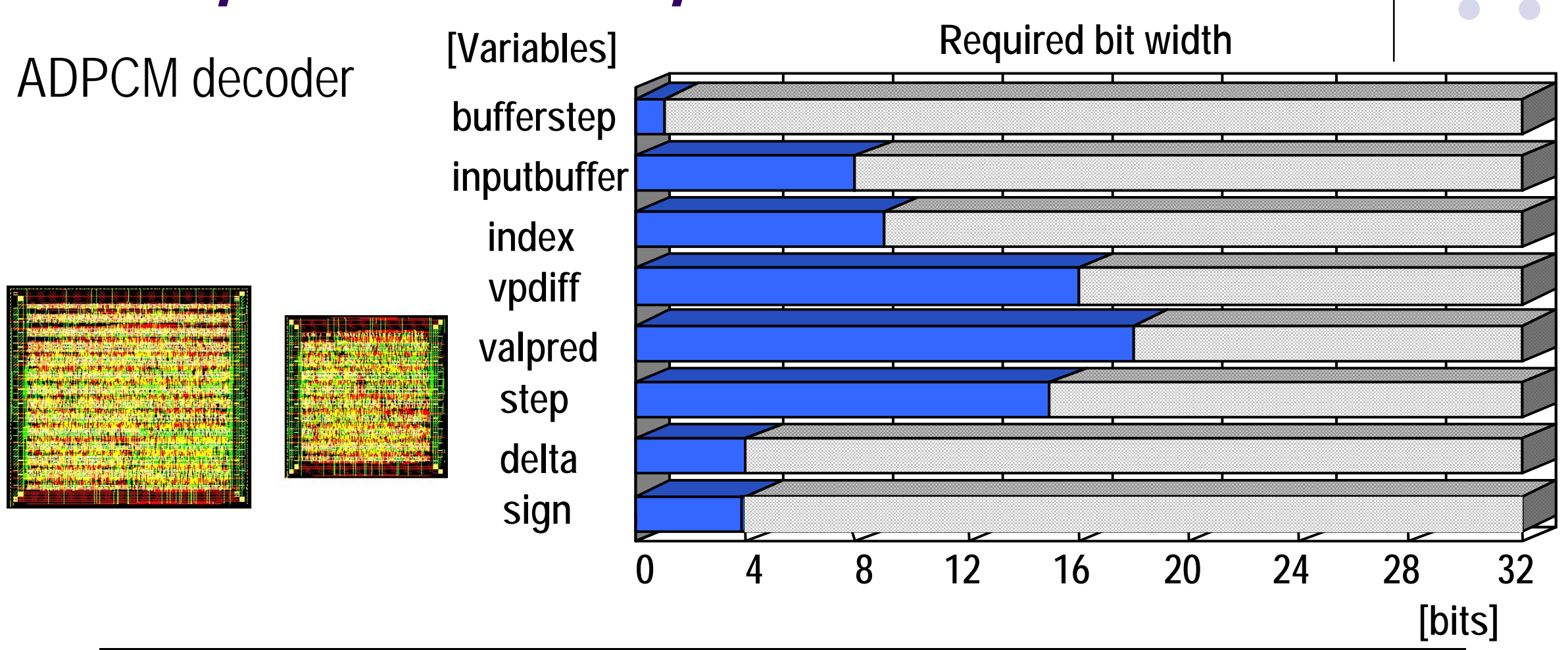

\begin{tabular}{|c|c|c|c|}
\hline & ADPCM32 & ADPCM18 & Reduction \\
\hline Area & $1220.8 \times 1196.0\left[\mathrm{um}^{2}\right]$ & $865.2 \times 865.2\left[\mathrm{um}^{2}\right]$ & $49 \%$ \\
\hline \#Cells & 1379 & 669 & $52 \%$ \\
\hline \#Transistors & 13006 & 5864 & $55 \%$ \\
\hline Energy & $367[\mathrm{~nJ}]$ & $239[\mathrm{~nJ}]$ & $35 \%$ \\
\hline 05. & \multicolumn{2}{|c}{ Process technology : NEL 0.5um }
\end{tabular}




\section{Datapath Width Adjustment for MPEG2 decoder}

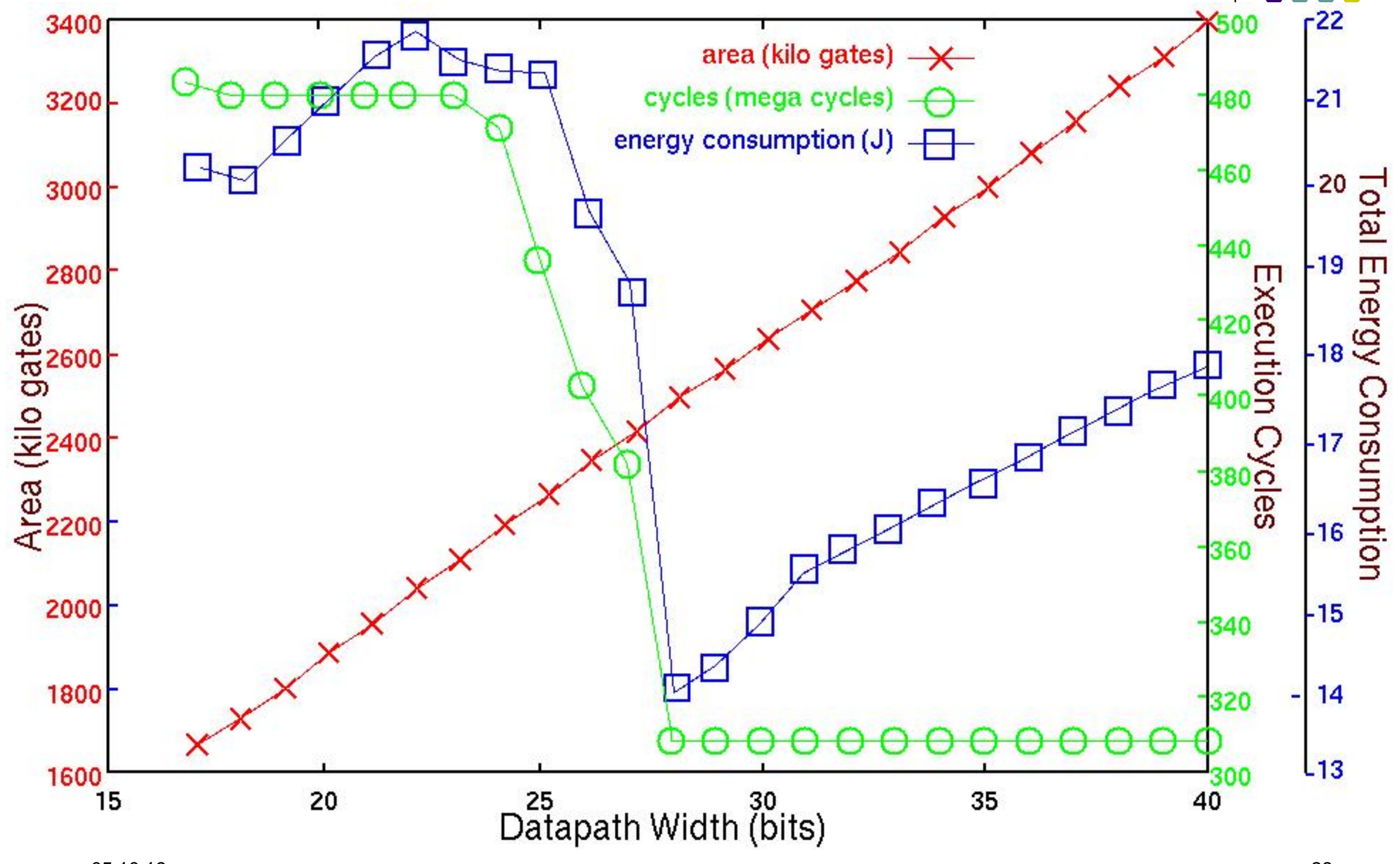




\section{Point 1}

- Datapath width adjustment can reduce power consumption without loss of performance and quality.

- Key techniques

- Variable size analysis

- Valen-C and Its compiler

- HW/SW co-design tools

- Softcore Processor

- Optimization of datapath width

- At most 50\% power reduction for practical applications 


\section{Datapath Width Adjustment}

$\square$ Quality-Driven Destign

- Adjust datapath width for required quality. -Reduce both upper and lower bits, if possible.

Program

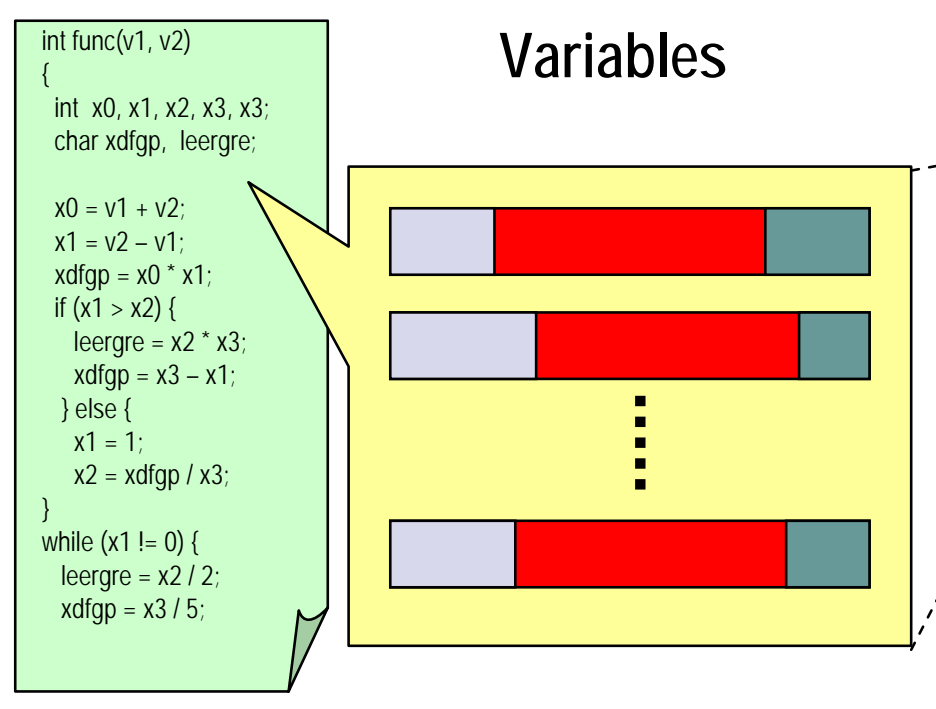

Datapath

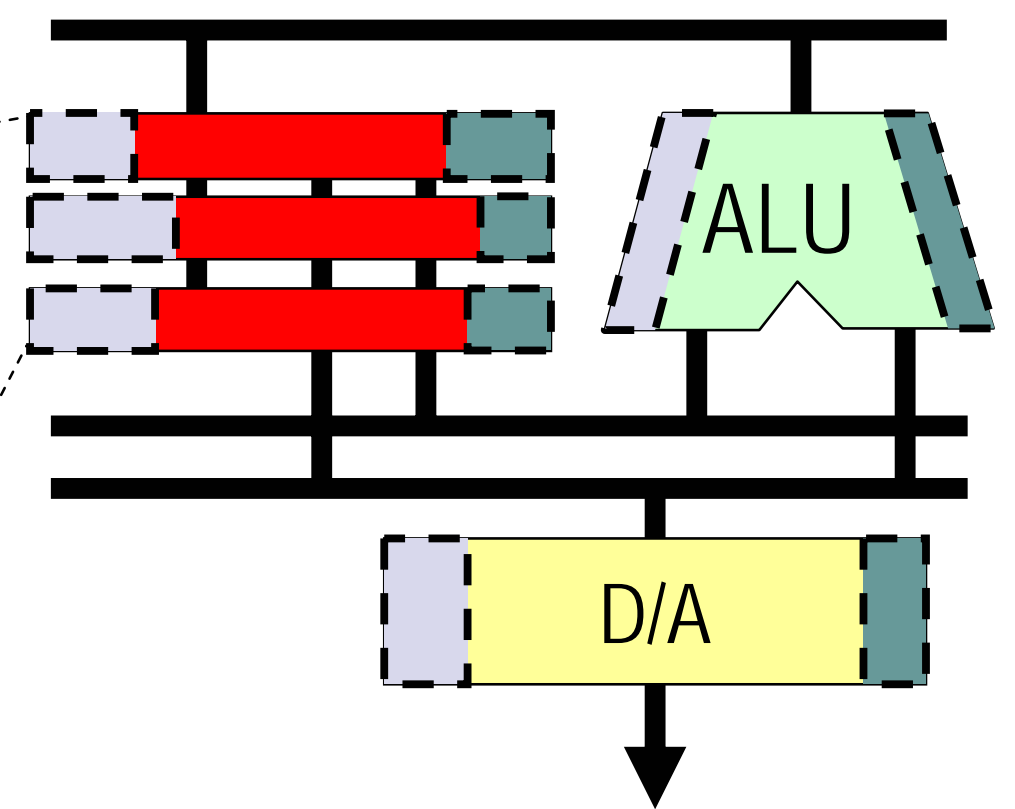

Output 


\section{Quality-Driven Design}

- Concepts

- Variety of output devices as human interfaces

- Mobile devices,

- low cost devices,

- high-quality devices,

- and ultra high-quality devices

- Prepare the least width of datapath for the requested accuracy of the computation
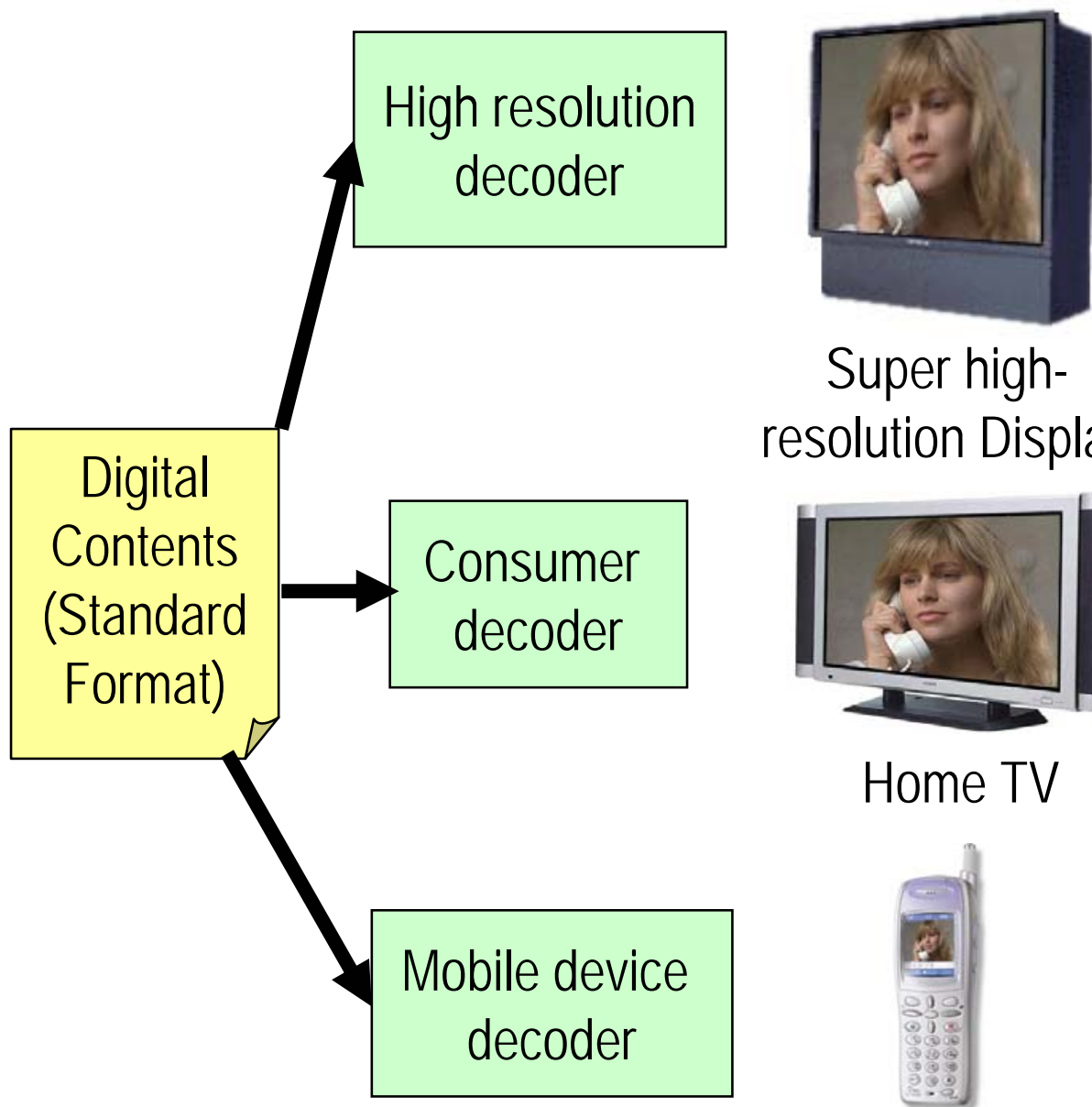

Super highresolution Display

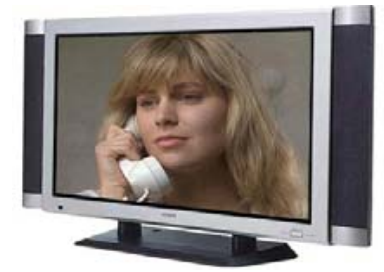

Home TV

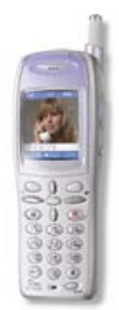




\section{Example of MPEG-2 Video Decoder}

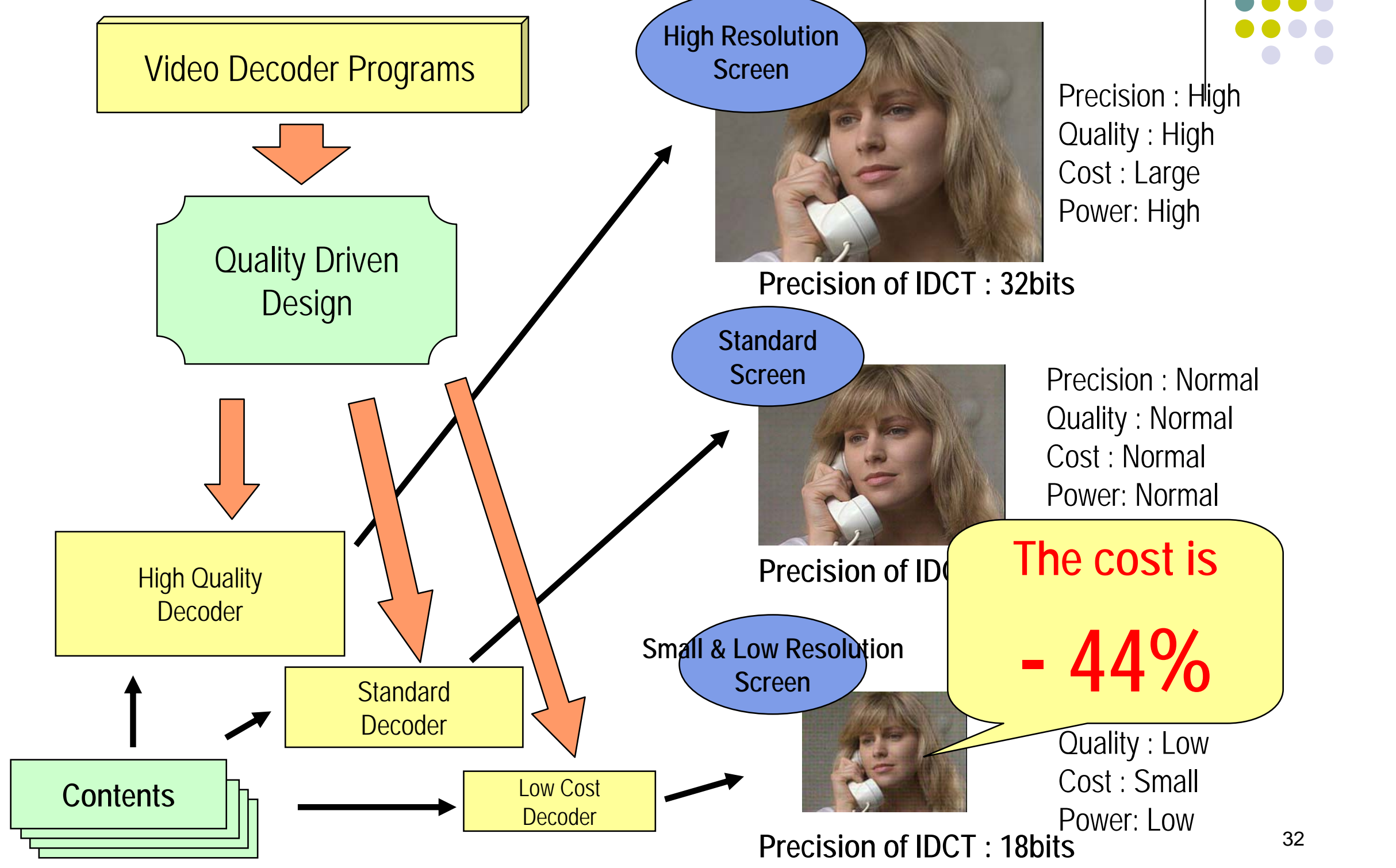




\section{Point 2}

- Quality-Driven Design

- Bridge between contents in standard formats and variety of output devices.

- Kinds of the product line approach

- From basic description and design parameter, we can get design with optimum datapath width automatically.

- New direction of HW/SW co-design 


\section{POWER OPTIMIZATION BY DATAPATH}

WIDTH ADJUSTMENT

- Introduction

- Datapath Width and Power Consumption

- Soft Core Processor

- Memory Width Optimization

- Shifted Computation

- Value-Based Clock Gating

- Conclusion 


\section{\# of Accesses of Variables}

\# of switching depends on \# of usages of data.
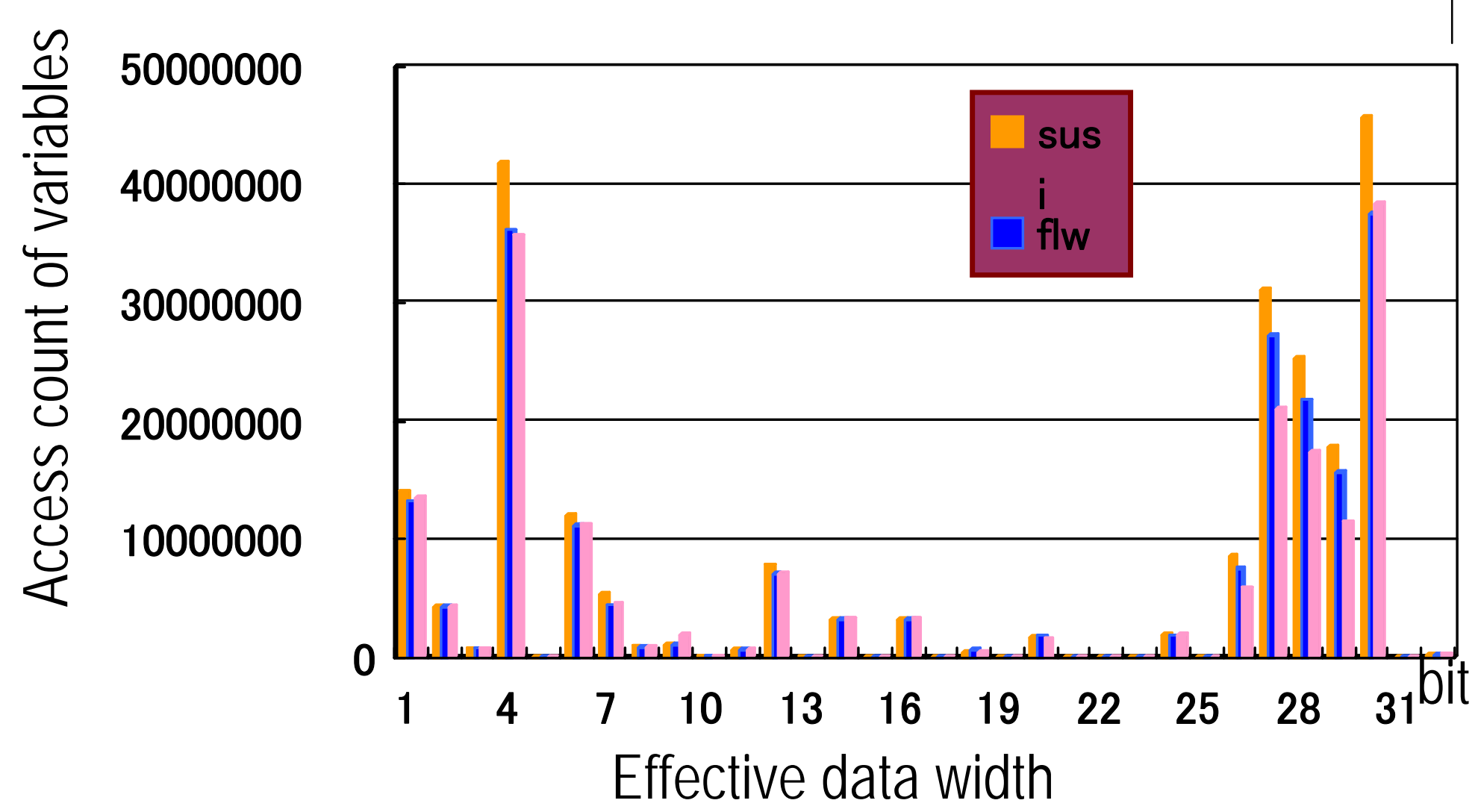

MPEG-2 video decoder 


\section{Memory Model}

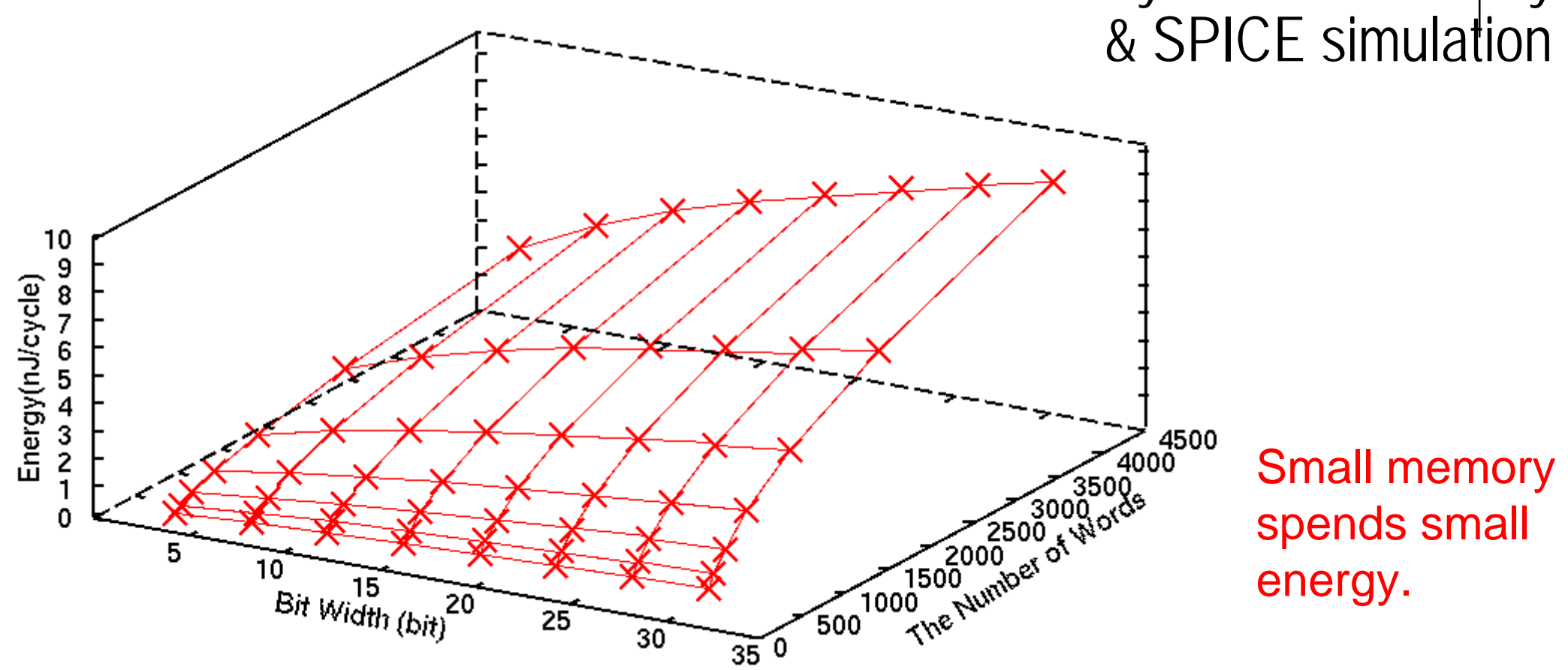

Bit width $x$ and word count $N_{\text {word }}$ V S. energy consumption of read access for SRAM

$$
\begin{array}{ll}
e_{r} \approx 24.9 \times x \times \sqrt{N_{\text {word }}}+56[\mathrm{pJ} / \text { cycle }] & \text { Read Access Energy } \\
e_{w} \approx 197 \times x \times \sqrt{N_{\text {word }}}+369[\mathrm{pJ} / \text { cycle }] & \text { Write Access Energy }
\end{array}
$$




\section{Multiple bit-width Memory Architecture}

Data Memory is divided into multiple modules with different bit-width Assign frequently accessed data into memory module with small words Assign small size data into memory module with small bit-width Optimize the data allocation to minimize total energy consumption

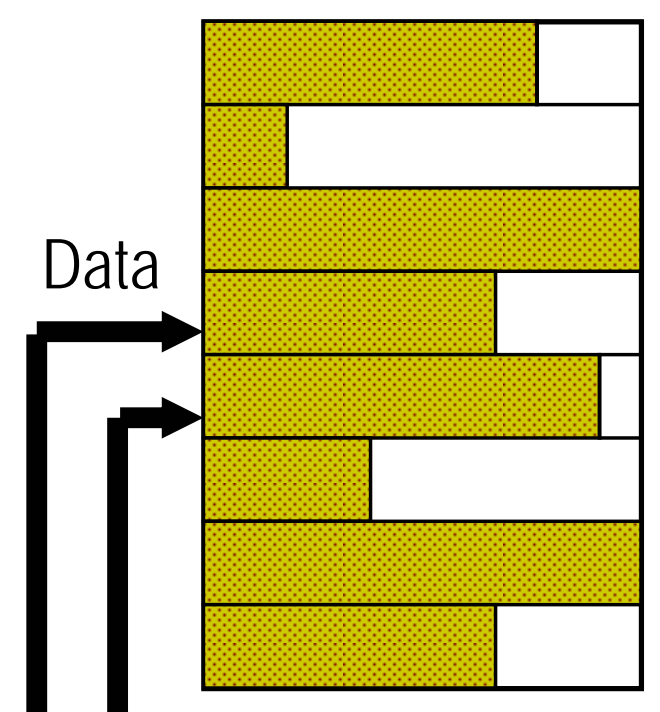

Addr

M.p.19olithic Memory
Optimize bit-width and \# of words.

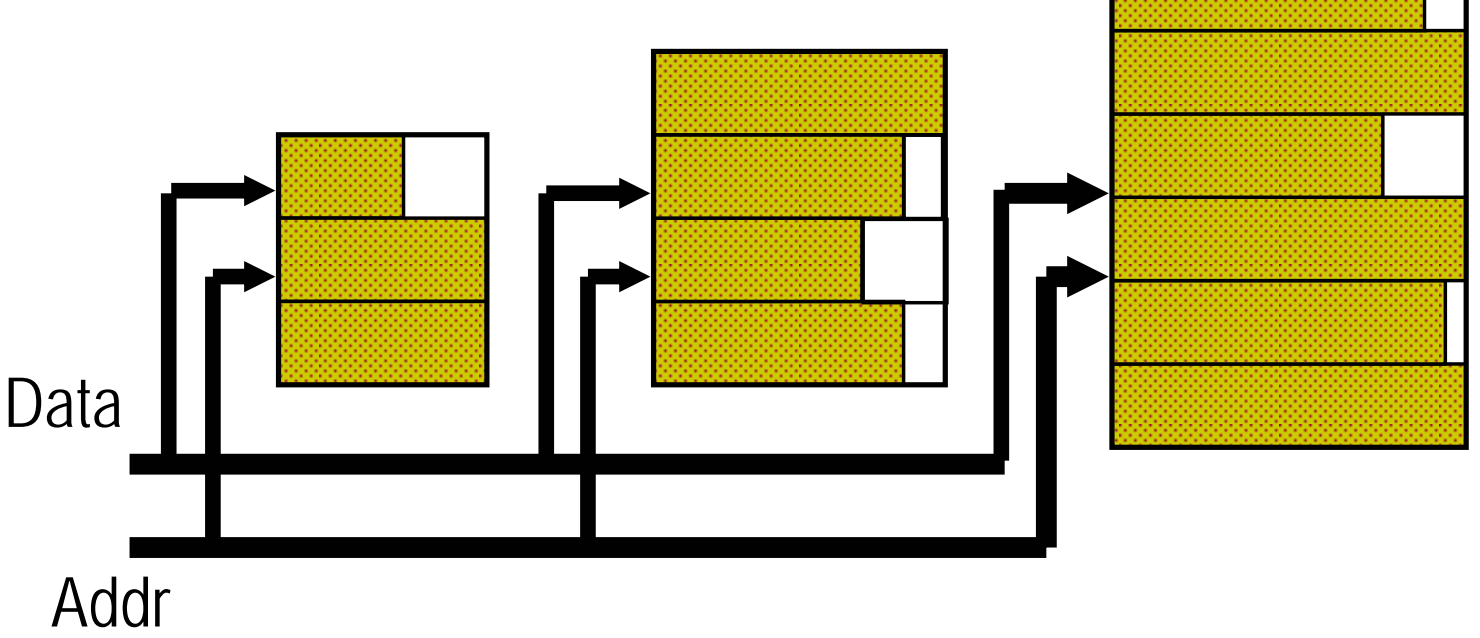

Multiple bit-width memory architecture 


\section{Experimental Results}

\begin{tabular}{|c|c|c|c|c|c|c|c|}
\hline \multirow[b]{2}{*}{ Applications } & \multirow{2}{*}{$\begin{array}{l}\text { Energy } \\
\text { (J) }\end{array}$} & \multicolumn{3}{|c|}{ Memory banking Technique } & \multicolumn{3}{|c|}{ Optimized Memory by VAbM } \\
\hline & & Configuration & $\operatorname{TEb}(\mathrm{J})$ & Sav. & Configuration & $\operatorname{TEm}(\mathrm{J})$ & Sav. \\
\hline Calculator & $\begin{array}{l}1.27 \\
\mathrm{~mJ}\end{array}$ & $\begin{array}{l}85 \text { rows } \\
154 \text { rows } \\
533 \text { rows }\end{array}$ & $\begin{array}{c}0.87 \\
\mathrm{~mJ}\end{array}$ & $31.5 \%$ & $\begin{array}{l}\text { 85rows X 8b } \\
\text { 154rows X 32b } \\
\text { 533rows X 32b }\end{array}$ & $\begin{array}{r}0.76 \\
\mathrm{~mJ}\end{array}$ & $40.2 \%$ \\
\hline Lempel-Ziv & 1.37 & $\begin{array}{l}\text { 830rows } \\
\text { 3rows } \\
\text { 1663rows }\end{array}$ & 0.89 & $35.0 \%$ & $\begin{array}{l}\text { 830rows X 13b } \\
\text { 3rows X 15b } \\
\text { 1663rows X 15b }\end{array}$ & 0.69 & $49.6 \%$ \\
\hline ADPCM & 1.63 & $\begin{array}{l}\text { 20rows } \\
\text { 16rows } \\
\text { 86rows }\end{array}$ & 1.10 & $32.5 \%$ & $\begin{array}{l}\text { 20rows X 10b } \\
\text { 16rows X 14b } \\
\text { 86rows X 19b }\end{array}$ & 0.80 & $50.9 \%$ \\
\hline MPEG2AAC & 1.05 & $\begin{array}{l}\text { 30rows } \\
2374 \text { rows } \\
\text { 4804rows }\end{array}$ & 0.39 & $62.9 \%$ & $\begin{array}{l}\text { 30rows X 20b } \\
\text { 2374rows X 32b } \\
\text { 4804rows X 32b }\end{array}$ & 0.37 & $64.8 \%$ \\
\hline MPEG2Video & $\begin{array}{c}145.1 \\
\mathrm{~kJ}\end{array}$ & $\begin{array}{l}\text { 26559rows } \\
\text { 26557rows } \\
\text { 28127rows }\end{array}$ & $\begin{array}{c}120.1 \\
\mathrm{~kJ}\end{array}$ & $17.2 \%$ & $\begin{array}{l}\text { 26559rows X 8b } \\
\text { 26557rows X 30b } \\
28127 \text { rows X 32b }\end{array}$ & $\begin{array}{c}105.2 \\
\mathrm{~kJ}\end{array}$ & $27.5 \%$ \\
\hline
\end{tabular}




\section{Point 3}

- Memory is an important target for bitwidth adjustment.

- Multiple memory banks with different bitwidth.

- Optimization of power consumption controlling \# of banks, bitwidth and \# of words.

- Up to $60 \%$ power reduction is possible. 


\section{POWER OPTIMIZATION BY DATAPATH WIDTH}

ADJUSTMENT

- Introduction

- Datapath Width and Power Consumption

- Soft Core Processor

- Memory Width Optimization

- Shifted Computation

- Value-Based Clock Gating

- Conclusion 


\section{Datapath Width Adjustment Techniques}

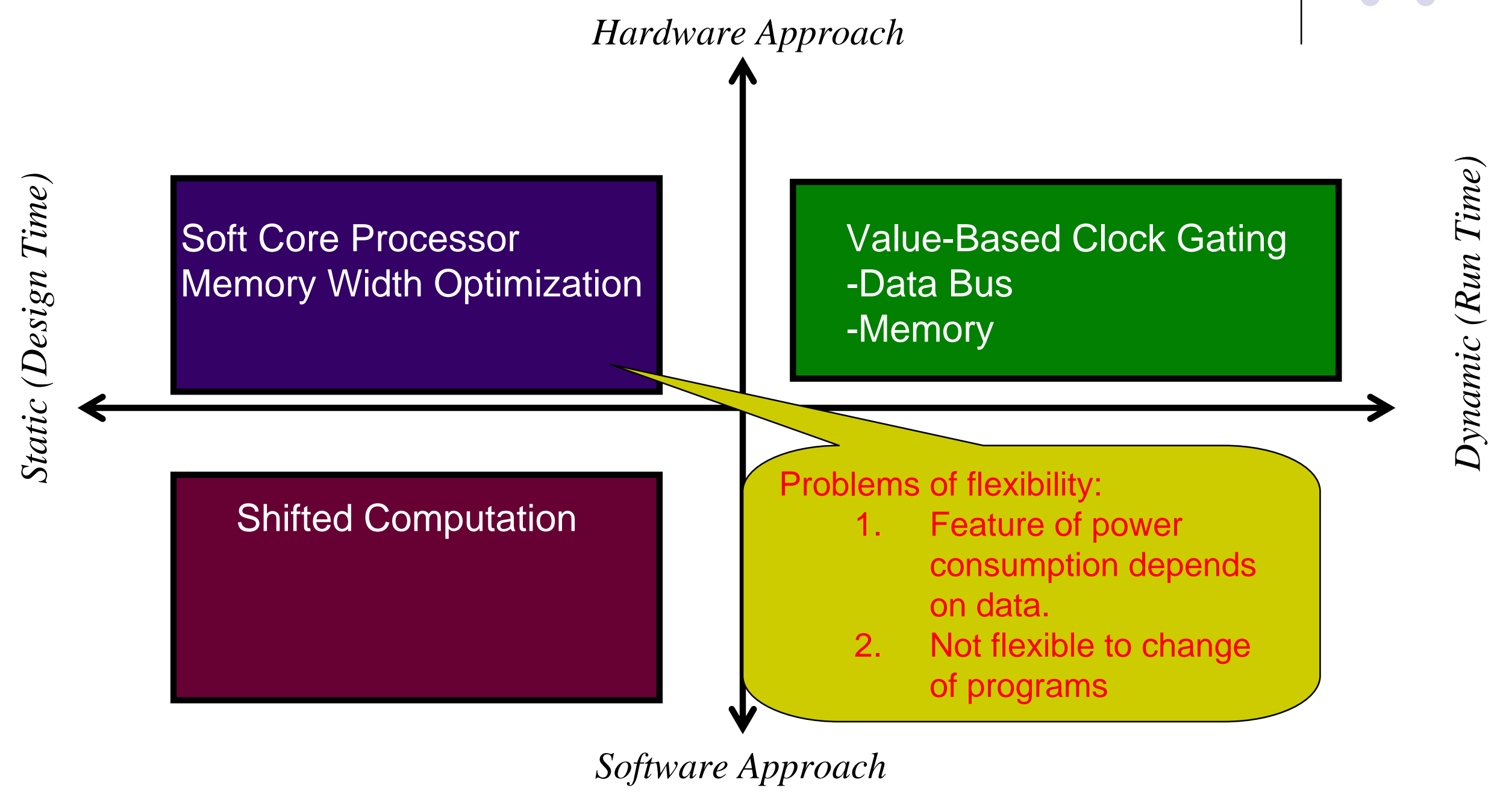




\section{Shifted Computation for Low-Precision}

- Software oriented approach

- Concepts

- For low precision computation, shifted computation is effective to reduce power consumption due to sign extension

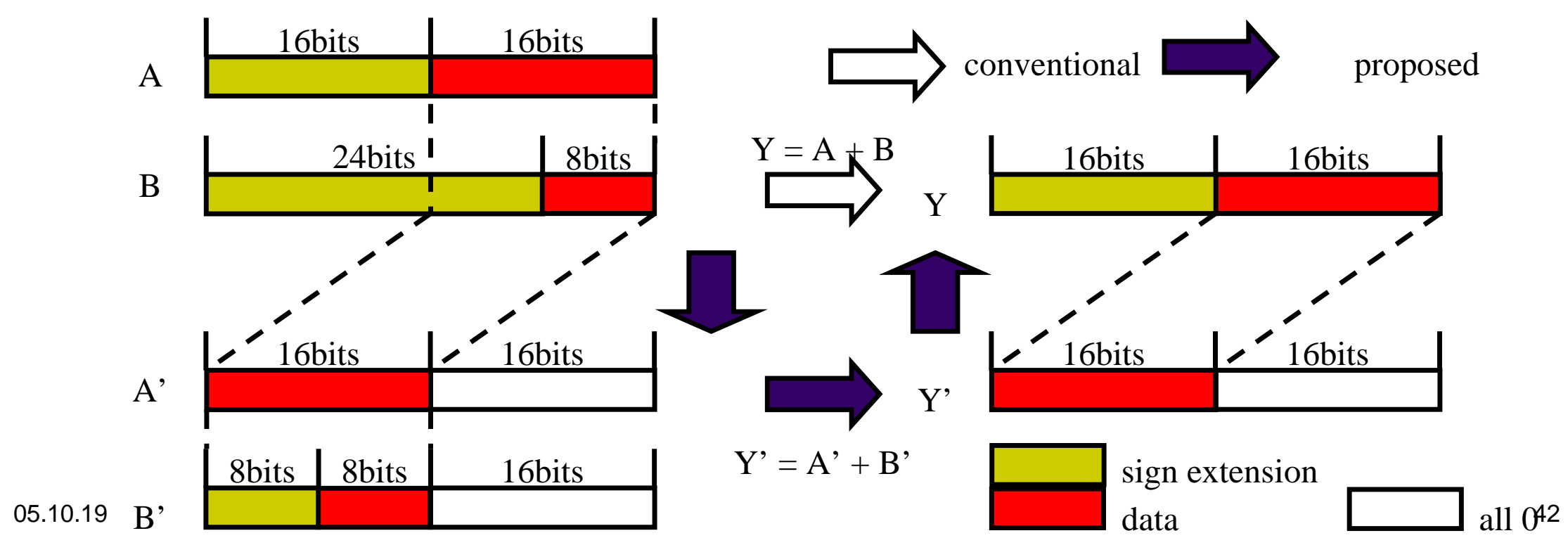




\section{Example of Shifted Computation for Low-Precision}

- ADPCM decoder

- Adding shift operations based on the required bitwidth analysis

- 32-bit CLA

Conventional

893 uW

Proposed

$551 \mathrm{uW}$

$38 \%$ saving

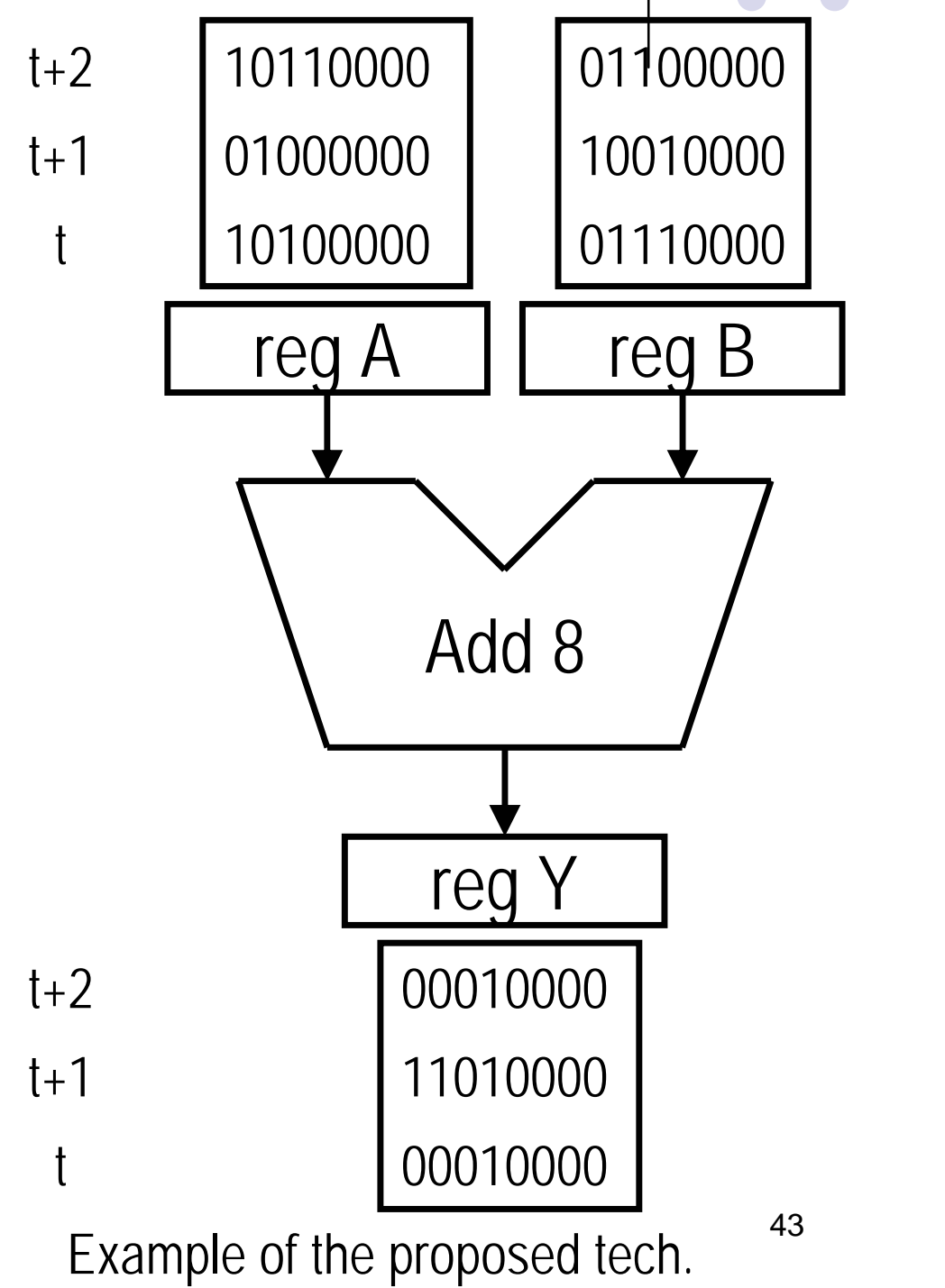




\section{Point 4}

- Software oriented approach

- No hardware change

- SW level optimization in compilation phase

- Overhead for shift opreation in performance and power (also instruction memory).

- On going research theme 


\section{POWER OPTIMIZATION BY DATAPATH WIDTH}

ADJUSTMENT

- Introduction

- Datapath Width and Power Consumption

- Soft Core Processor

- Memory Width Optimization

- Shifted Computation

- Value-Based Clock Gating

- Conclusion 


\section{Datapath Width Adjustment Techniques}

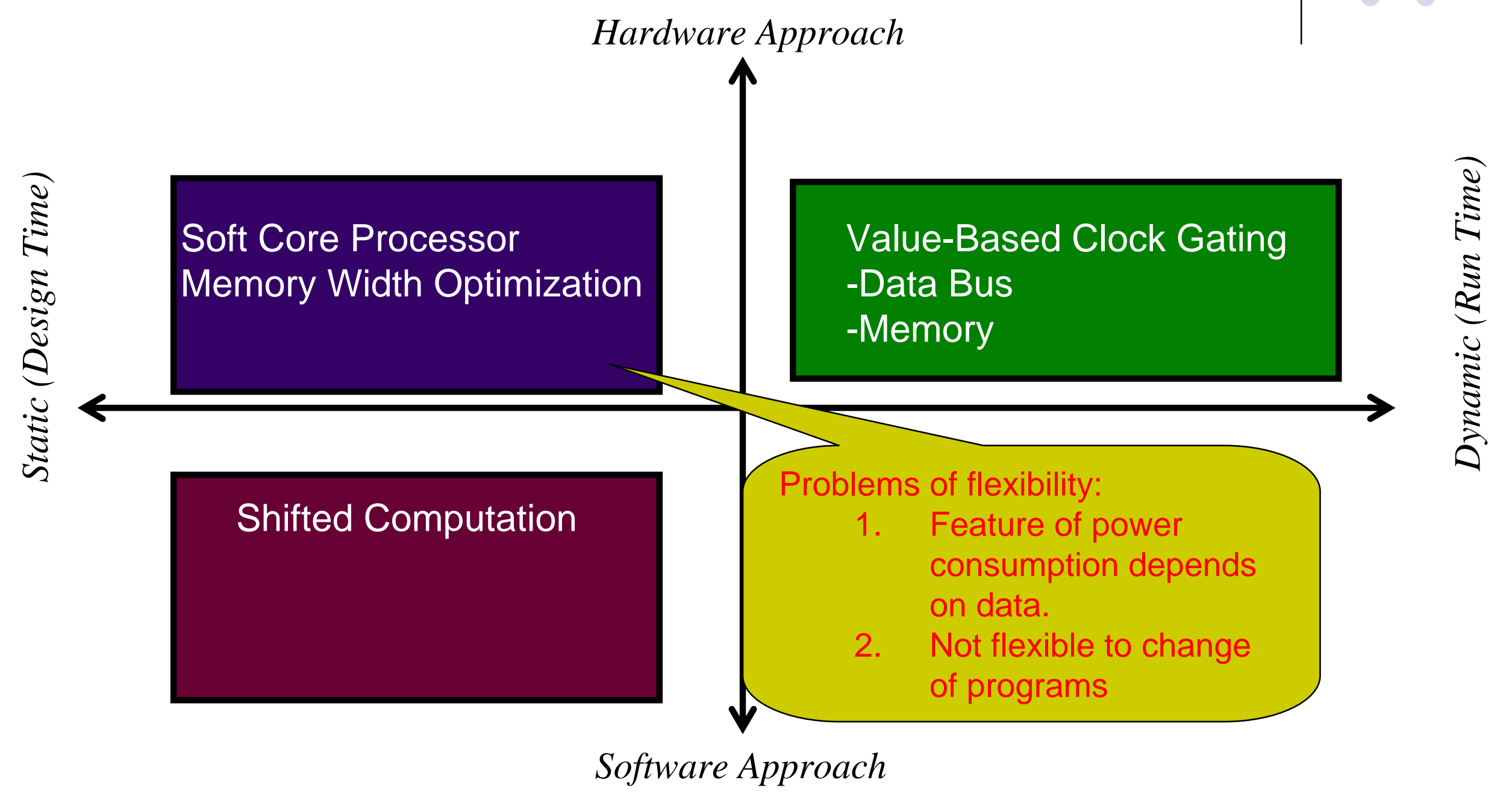




\section{Active Bits in Computation}

- Active bits: the bits expressing the value of data except sign extension.

$0000000{\underline{111110001_{2}}}_{2}$

- For a variable $x$, active bits of instances are different from each other.

- Variable size analysis gives the worst case of the length of active bits.

- How to identify the active bits? 


\section{How to decide Active Data Bitwidth}

The case of 16 bits data width

- Method 1) remains after deleting the continuous "0" in upper bits

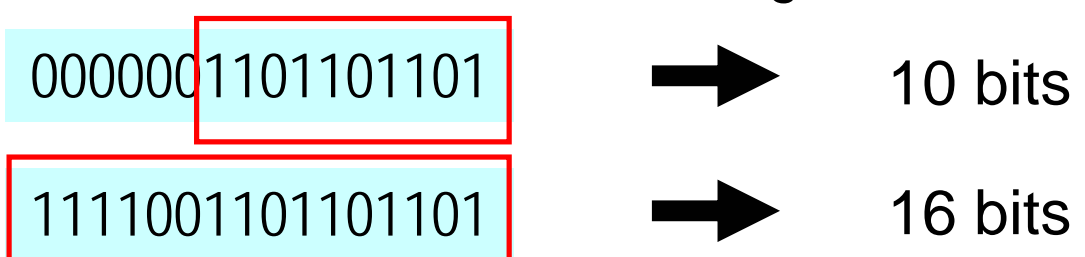

- Method 2) remains afier deleting the continuous "1" in upper bits

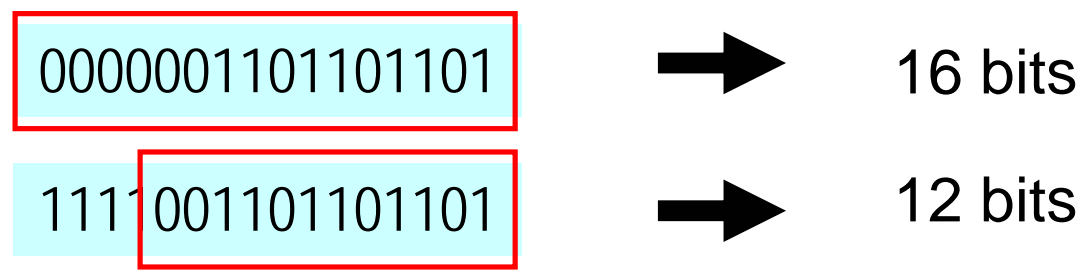

- Method 3) remains after changing the continuous "0" to only one "0", or remains after changing the continuous "1" to only one " 1 "

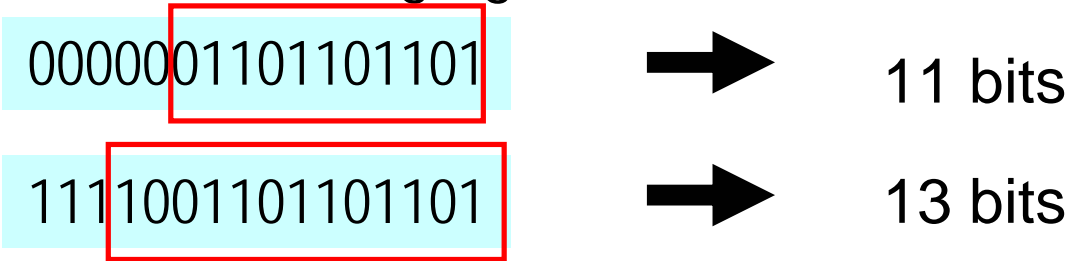




\section{Value-Based Clock Gating}

- Concepts

- Since small number can be expressed by using small number of bits, gating remaining redundant bits for reducing power consumption

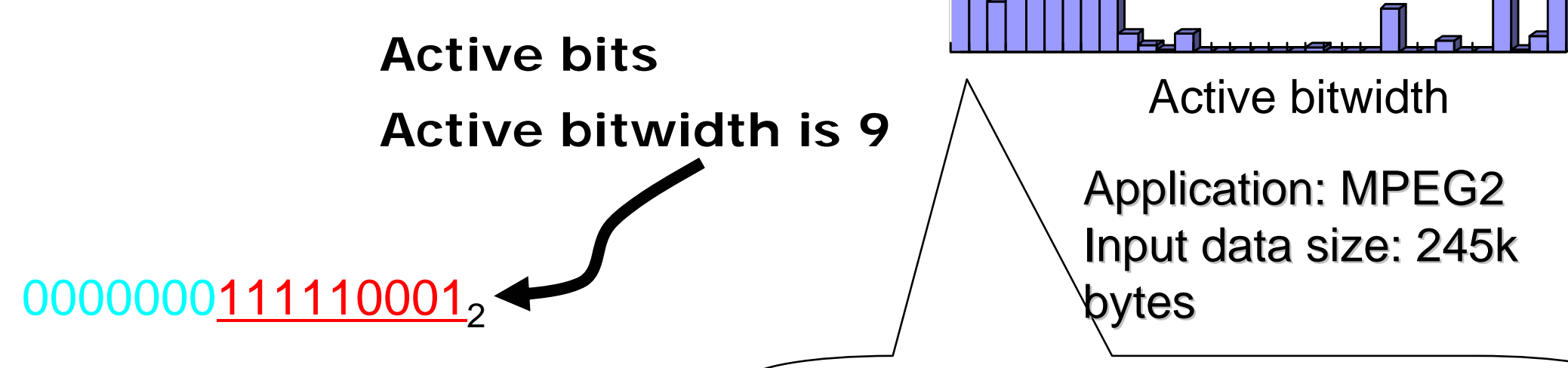

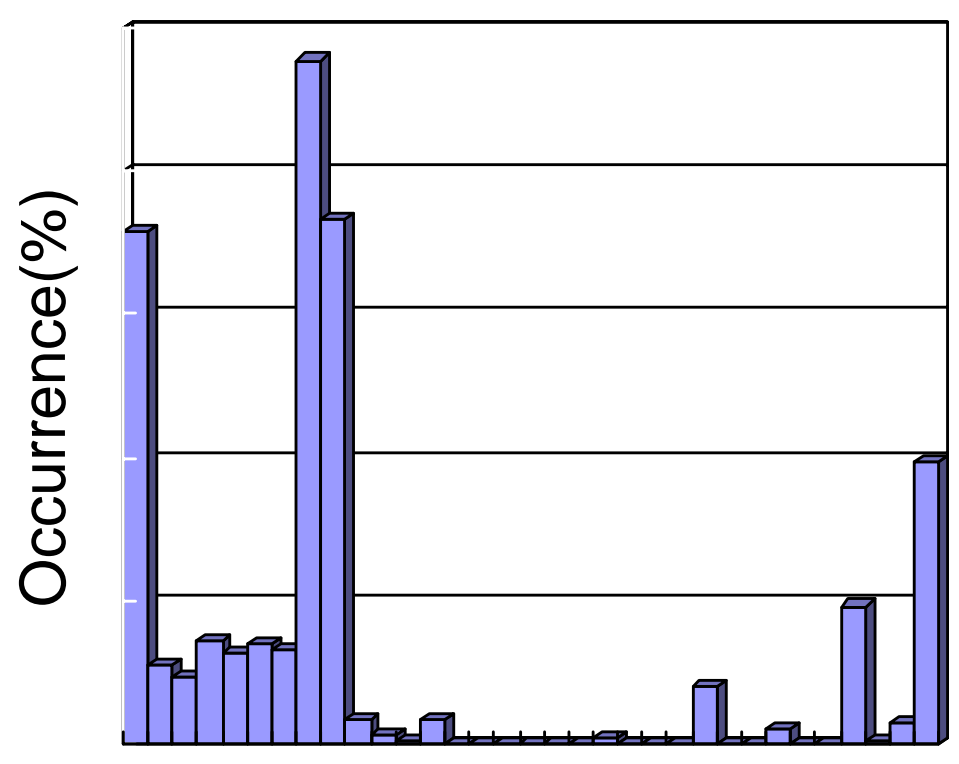

About $80 \%$ of active bitwidth of data is 8 or less 


\section{Example of \\ Value-Based Clock Gating}

\section{6-bit data bus}

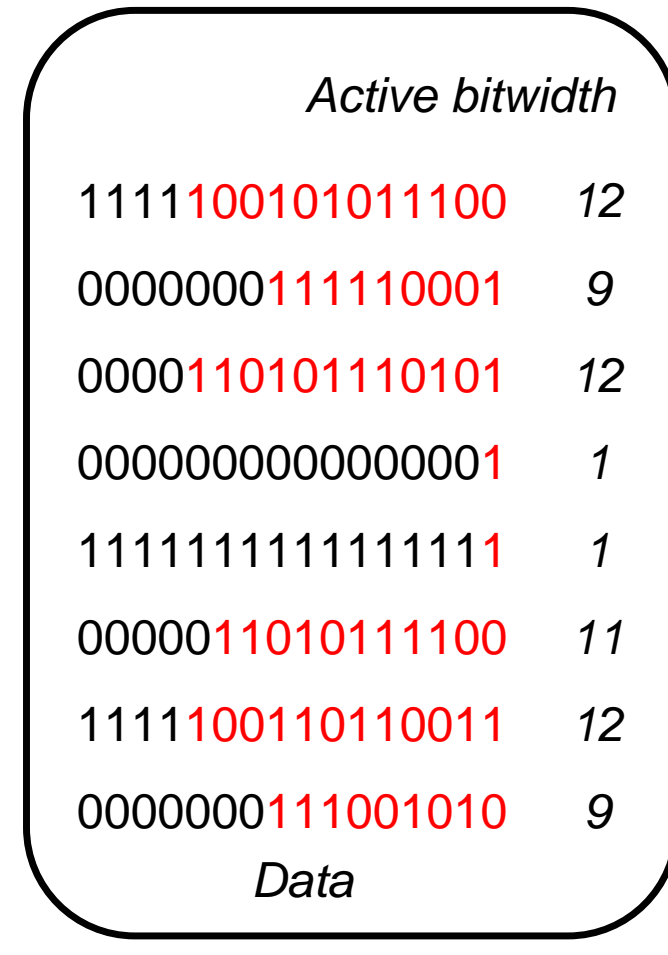

\# of Switching 67

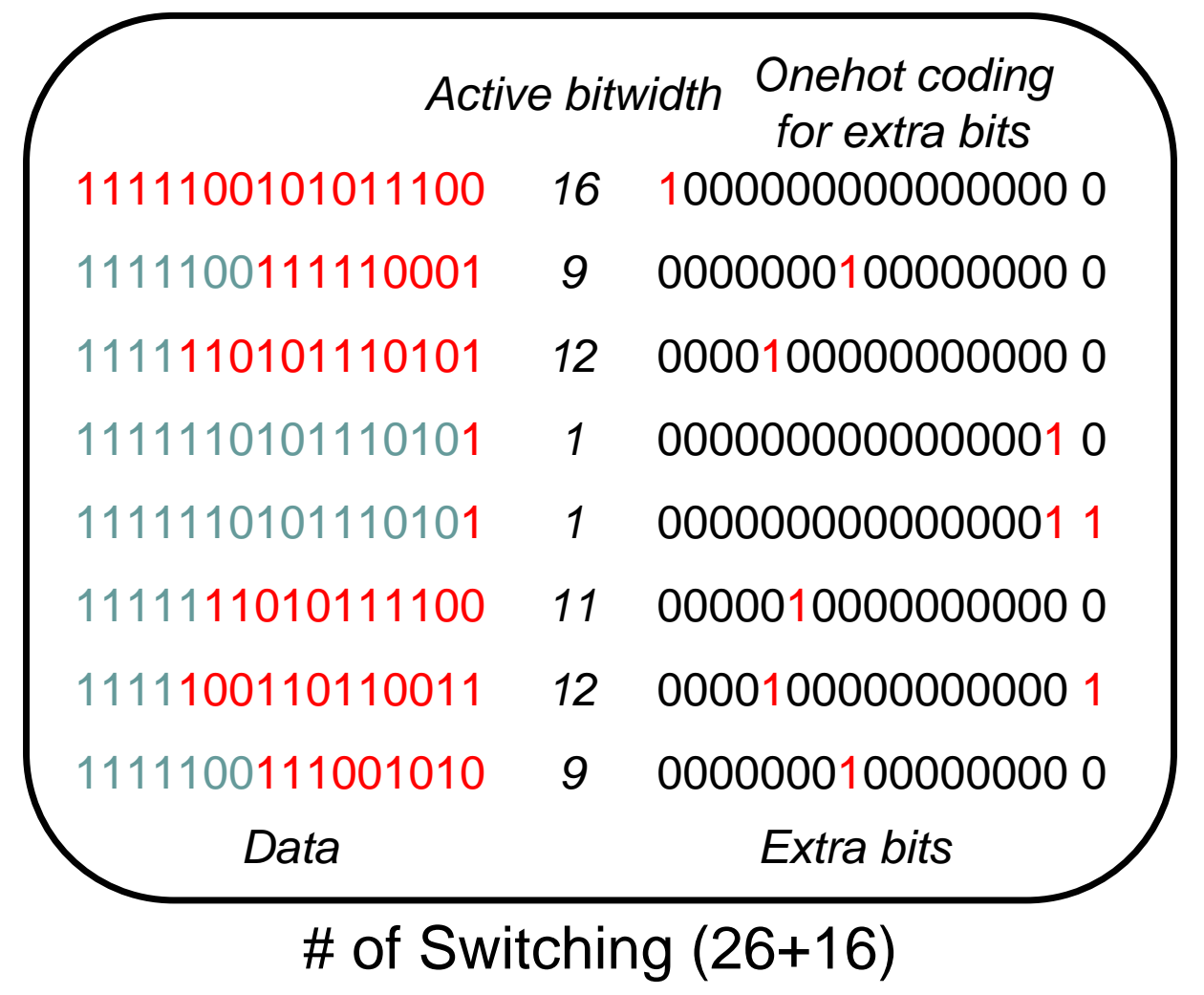

Encoded data using our approach 
Transition reduction ratio(\%): in case of onehot codin

\begin{tabular}{|c|c|c|c|c|c|c|}
\hline & \multicolumn{6}{|c|}{$\mathrm{N}_{\mathrm{seg}}$} \\
\hline & 32 & 16 & 8 & 4 & 2 & 1 \\
\hline \multicolumn{7}{|l|}{ mpeg2 } \\
\hline (116k) & -37.1 & -35.5 & -34.2 & -31.8 & -22.9 & -6.48 \\
\hline (245k) & -45.6 & -44.1 & -42.7 & -40.3 & -27.0 & -5.19 \\
\hline (649k) & -34.4 & -32.1 & -31.0 & -28.0 & -21.9 & -6.86 \\
\hline go & -33.8 & -28.8 & -28.8 & -38.9 & -30.5 & -20.3 \\
\hline gzip & -10.5 & -12.0 & -9.19 & -7.63 & -1.94 & -1.58 \\
\hline bzip & -17.8 & -18.5 & -19.1 & -18.5 & -19.2 & -29.6 \\
\hline perl & -14.5 & -13.8 & -11.8 & -11.5 & -3.67 & -5.25 \\
\hline mesa & -19.0 & -17.1 & -16.1 & -13.6 & -10.8 & -15.9 \\
\hline wupwise & -18.0 & -16.6 & -15.6 & -16.2 & -9.71 & -5.02 \\
\hline Ave. & -22.7 & -21.6 & -20.5 & -20.9 & -14.7 & -11.8 \\
\hline
\end{tabular}


Transition reduction ratio(\%): in case of binary coding

\begin{tabular}{|l|r|r|r|r|r|}
\hline \multirow{2}{*}{ App. } & \multicolumn{6}{|c|}{$N_{\text {seg }}$} \\
\cline { 2 - 6 } & \multicolumn{7}{|c|}{32} & 16 & 8 & 4 & 2 \\
$(116 k)$ & -30.0 & -35.4 & -37.9 & -38.6 & -26.9 \\
\cline { 2 - 6 }$(245 k)$ & -40.7 & -44.8 & -46.5 & -45.3 & -30.9 \\
\cline { 2 - 6 }$(649 k)$ & -26.7 & -31.3 & -34.4 & -35.8 & -26.0 \\
\hline go & -23.7 & -28.8 & -39.0 & -49.1 & -20.3 \\
\hline gzip & -8.83 & -11.6 & -12.7 & -12.2 & -8.13 \\
\hline bzip2 & -7.19 & -13.1 & -19.1 & -25.6 & -31.1 \\
\hline perl & -13.3 & -10.8 & -14.2 & -16.7 & -12.0 \\
\hline mesa & -6.75 & -9.30 & -14.5 & -20.0 & -22.1 \\
\hline wupwise & -12.3 & -13.9 & -17.7 & -18.7 & -15.2 \\
\hline Ave. & -16.1 & -18.9 & -23.1 & -26.8 & -20.0 \\
\hline
\end{tabular}




\section{Applicaton to Memory}

- Memory Vertical Partitioning

Active Data Bitwidth 13 bits

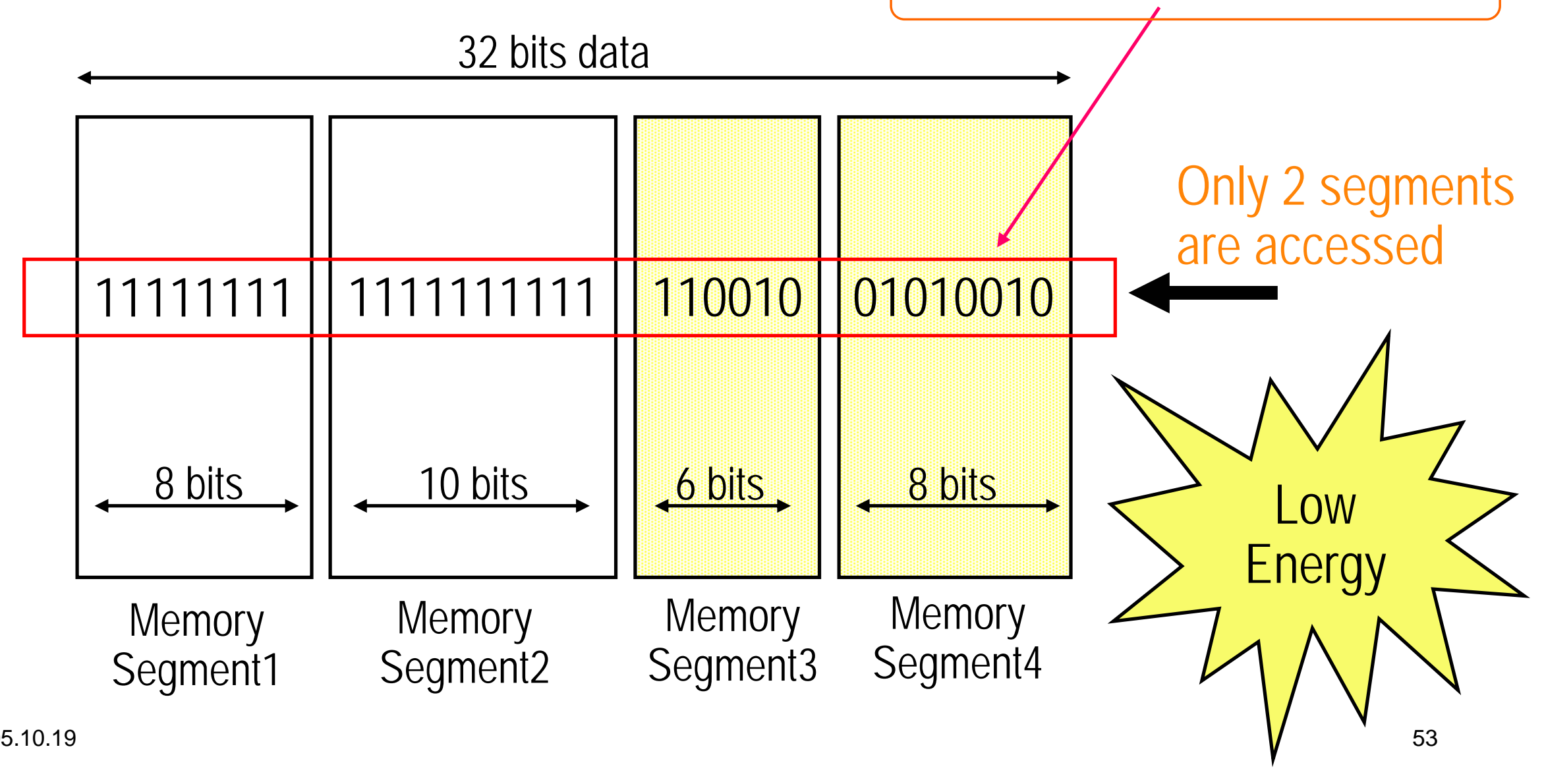




\section{Memory Partitioning Example}

s!neg

.

000

1000

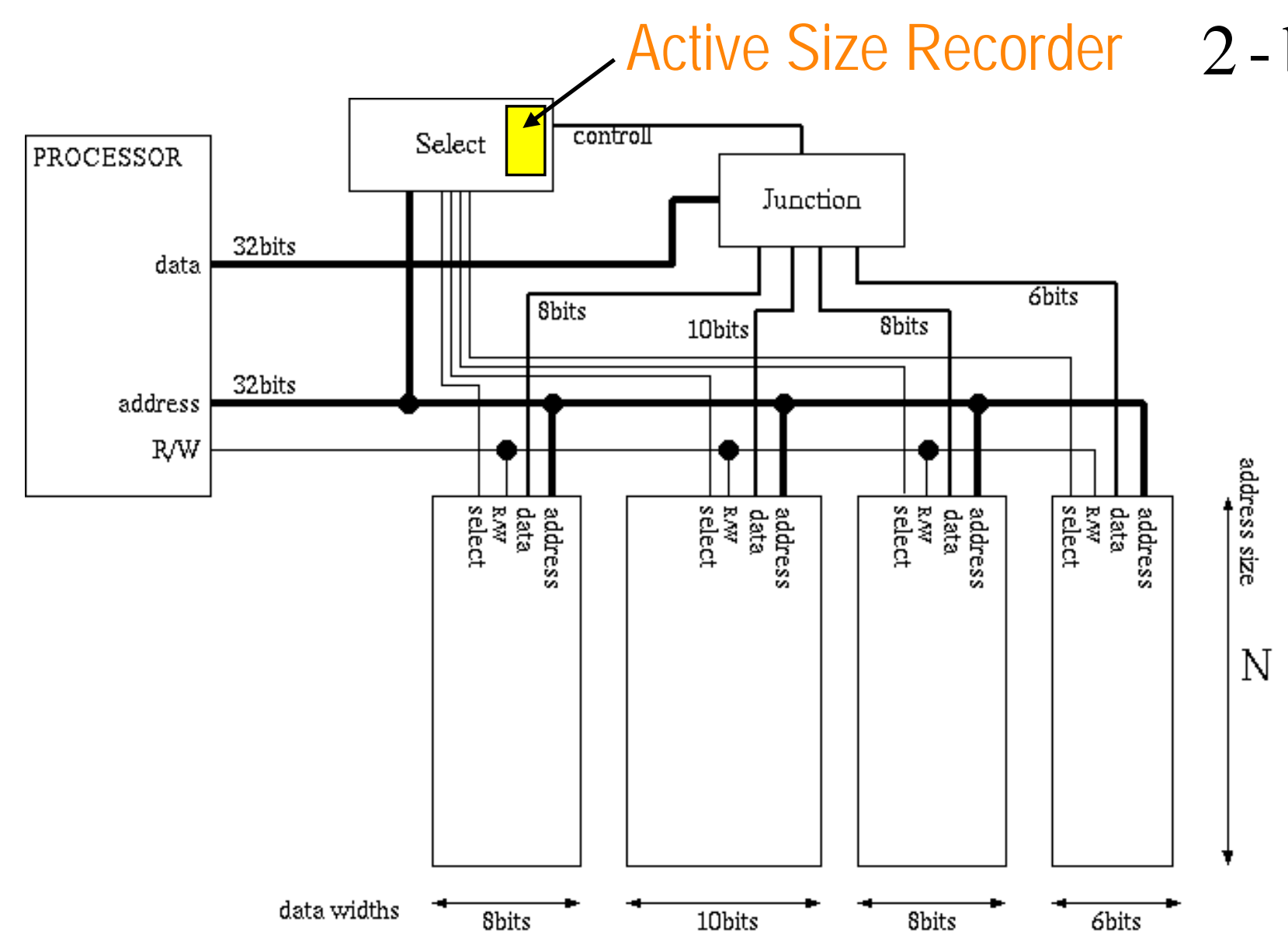




\section{Experimental Results}

\begin{tabular}{|c|c|c|c|c|c|c|}
\hline \multirow{2}{*}{$\begin{array}{l}\text { Mpeg2play } \\
\qquad N_{\text {word }} 302834\end{array}$} & \multicolumn{2}{|l|}{ Memory } & \multicolumn{2}{|c|}{ Active Size Recorder } & \multirow{2}{*}{$\begin{array}{l}\text { Total } \\
\text { Energy }\end{array}$} & \multirow{5}{*}{$\begin{array}{l}b_{1}=8 \\
b_{2}=8 \\
b_{3}=8 \\
b_{4}=8\end{array}$} \\
\hline & Read & Write & Read & Write & & \\
\hline Our Technique & $0.75 \mathrm{~J}$ & $0.59 \mathrm{~J}$ & $0.14 \mathrm{~J}$ & $0.74 \mathrm{~J}$ & $2.22 \mathrm{~J}$ & \\
\hline $\begin{array}{l}\text { Monolithic } \\
\text { Memory }\end{array}$ & $2.3 \mathrm{~J}$ & $11.8 \mathrm{~J}$ & & & $14.1 \mathrm{~J}$ & \\
\hline Memory Banking & $1.08 \mathrm{~J}$ & $5.43 \mathrm{~J}$ & & & $6.51 \mathrm{~J}$ & \\
\hline
\end{tabular}

\begin{tabular}{|c|c|c|c|c|c|}
\hline \multirow{2}{*}{$\begin{array}{l}\text { IJPEG } \\
\qquad N_{\text {word }} 917987\end{array}$} & \multicolumn{2}{|l|}{ Memory } & \multicolumn{2}{|c|}{ Active Size Recorder } & \multirow{2}{*}{$\begin{array}{l}\text { Total } \\
\text { Energy }\end{array}$} \\
\hline & Read & Write & Read & Write & \\
\hline Our Technique & $3.16 \mathrm{~J}$ & $4.36 \mathrm{~J}$ & $0.37 \mathrm{~J}$ & $0.76 \mathrm{~J}$ & $8.65 \mathrm{~J}$ \\
\hline $\begin{array}{l}\text { Monolithic } \\
\text { Memory }\end{array}$ & $5.94 \mathrm{~J}$ & $12.17 \mathrm{~J}$ & & & $18.11 \mathrm{~J}$ \\
\hline Memory Banking & $4.58 \mathrm{~J}$ & $5.28 \mathrm{~J}$ & & & $9.86 \mathrm{~J}$ \\
\hline
\end{tabular}




\section{Point 5}

- Active data bitwidth is a dynamic approach for datapath adjustment.

- Overheads

- How to recognize active bits.

- How to keep the information of the active bits.

- How to control switching.

- No effects in SW level.

- Applicable for Logic, Processors and Memories. 


\section{POWER OPTIMIZATION BY DATAPATH WIDTH}

ADJUSTMENT

- Introduction

- Datapath Width and Power Consumption

- Soft Core Processor

- Memory Width Optimization

- Shifted Computation

- Value-Based Clock Gating

- Conclusion 


\section{Datapath Width Adjustment Techniques}

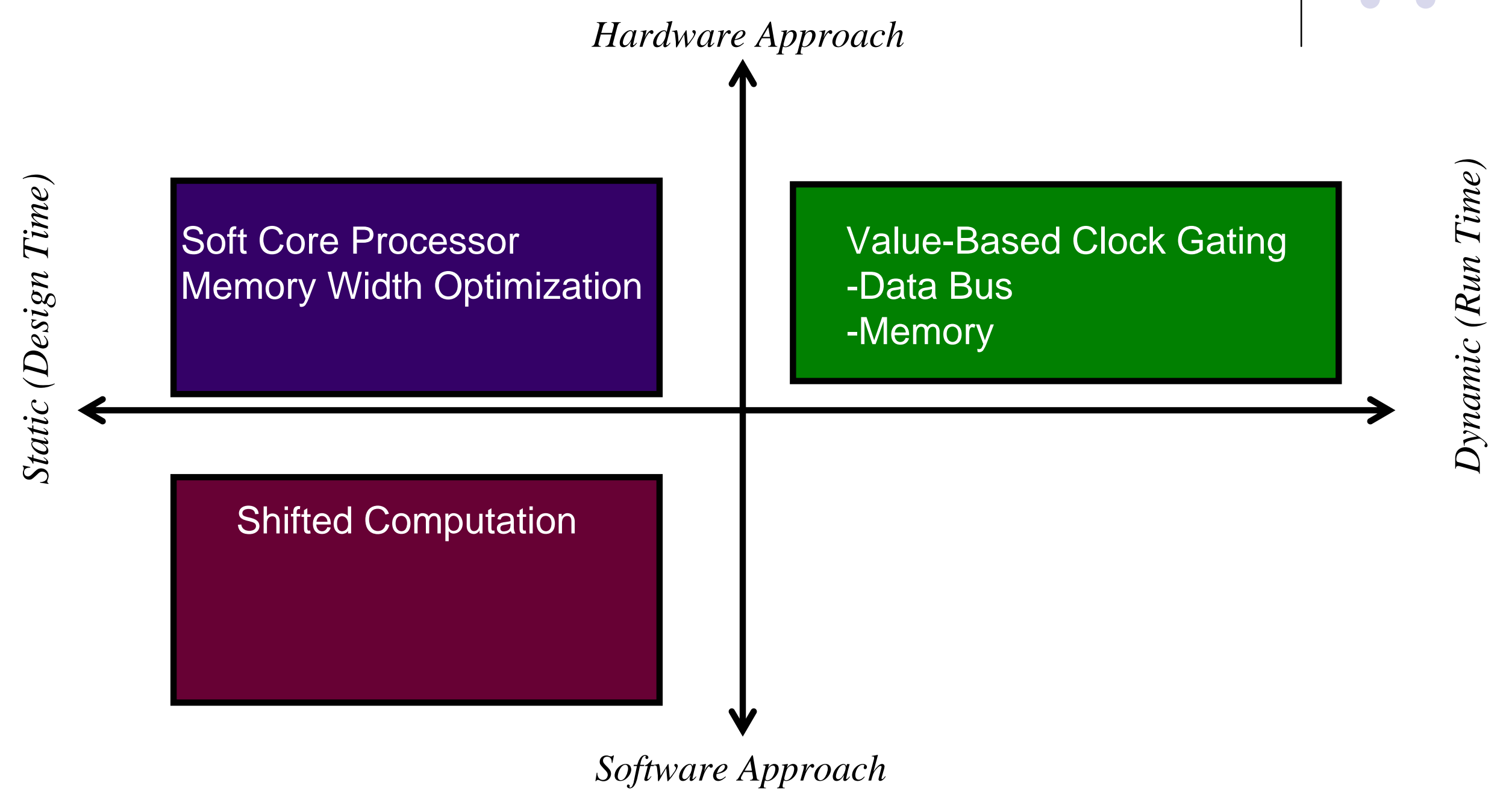




\section{Datapath Width Adjustment}

- Datapth adjustment is an important method for power and/or energy reduction.

- Various approaches are possible to apply for logic circuits, processors, memories and software.

- It is a new direction of reconstruction of compiler and processor architecture technology.

- SoC requires clear and simple principles for system level optimization. Through the researches on the datapath adjustment, we can find a hints of system level optimization approaches. 


\section{References}

- General

- Takanori Okuma, Tohru Ishihara, and Hiroto Yasuura, "Software Energy Reduction Techniques for Variable Voltage Prodessors", IEEE Design \& Test of Computers March-April 2001, Vol.18, No.2, pp.31-41, Apr. 2001.

- Soft Core Processor

- Hiroto Yasuura and Hiroyuki Tomiyama, “Power Optimization by Datapath Width Adjustment", In Massoud Pedram and Jan M. Rabaey, editors, Power Aware Design Methodologies, chapter 7, pp.181-199, Kluwer Academic Publishers, Jun. 2002.

- Yun Cao and Hiroto Yasuura, "A System-level Energy Minimization Approach Using Datapath Width Optimization", IEICE Technical Report, VLD2000-142, ICD2000-218(2001-03), pp.49-54, Mar. 2001.

- Quality Driven Design

- Y Yun Cao and Hiroto Yasuura, "Video Quality Modeling for Quality-driven Design", The 10th Workshop on System And System Integration of Mixed Technologies(SASIMI 2001), pp.86-92, Oct. 2001.

- Yun Cao and Hiroto Yasuura, "Quality-Driven Design for Video Applications", IEICE Transactions on Fundamentals of Electronics, Vol.E85-A, No.12, pp.2568-2576, Dec. 2002.

- Memory Width Optimization

- Y Yun Cao and Hiroto Yasuura, “'A Low-Energy Memory Design Technique Based on Variable Analysis for Application-Specific Systems", ACM the 12th Great Lakes Symposium on VLSI (GLSVLSI2002), Apr. 2002.

- Y Yun Cao and Hiroto Yasuura, "Memory Organization for Low-Energy Processor-Based Application-Specific Systems", IEICE Transactions on Electron, Vol.J83-C, No.8, pp.1616-1624, Aug. 2002.

- Value-Based Clock Gating

- Masanori Muroyama, Akihiko Hyodo, Takanori Okuma, and Hiroto Yasuura, “'A Power Reduction Scheme for Data Buses by Dynamic Detection of Active Bits", Proceedings of Euromicro Symposium on Digital System Design -Architectures, Methods and Tools-(DSD2003), pp.408-415, Sep. 2003.

- Masanori Muroyama, Akihiko Hyodo, Takanori Okuma, and Hiroto Yasuura, “A Power Reduction Scheme for Data Buses by Dynamic Detection of Active Bits", IEICE Transactions on Electronics, Vol.E87-C, No.4, pp.598-605, Apr. 2004.

- Takanori Okuma, Yun Cao, Masanori Muroyama, and Hiroto Yasuura, "Reducing Access Energy of On-Chip Data Memory Considering Active Data Bitwidth", Proc. of International Symposium on Low Power Electronics and Design(ISLPED'02), pp.88-91, Aug. 2002. 

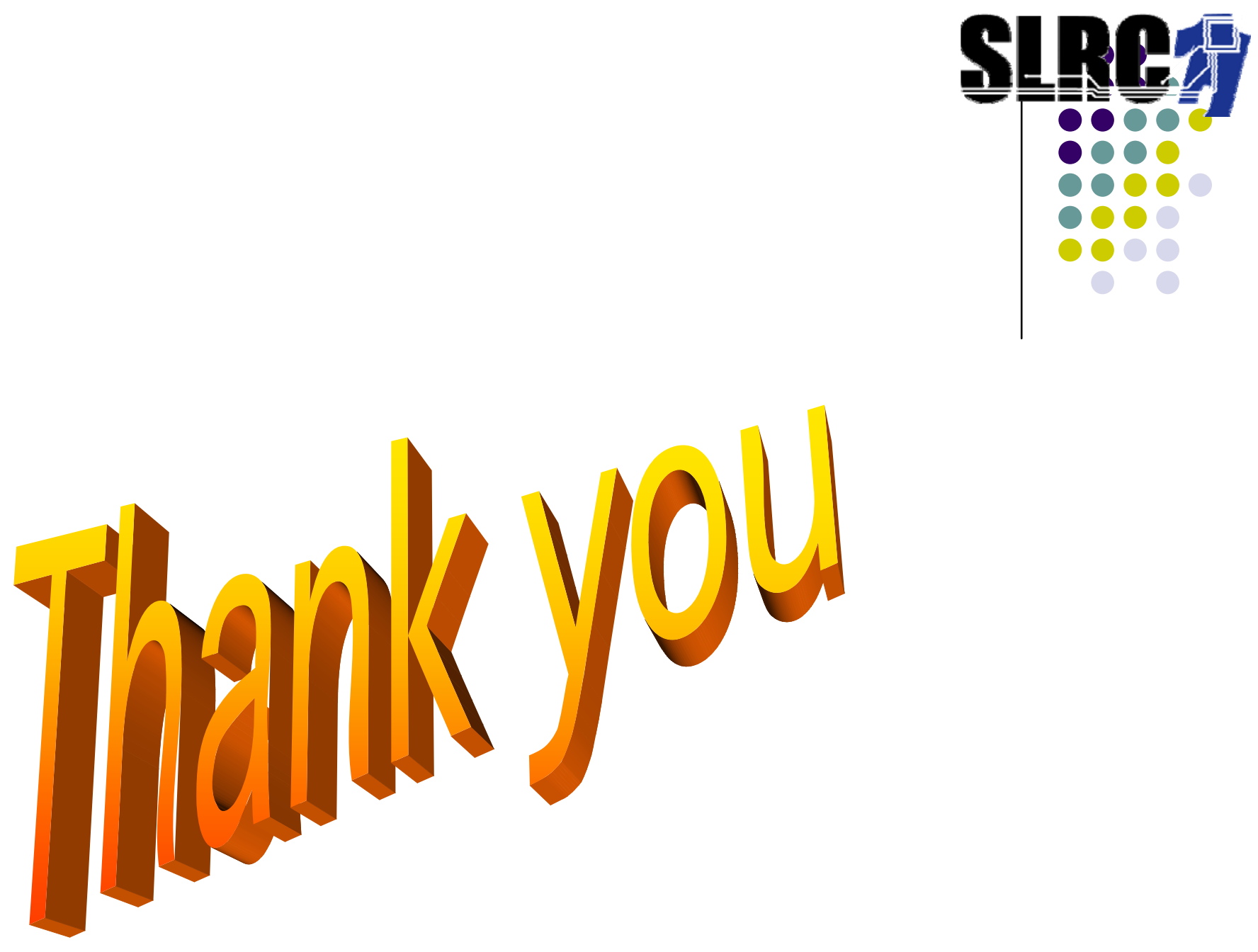